\title{
Aggregating Audit Evidence with a Markov Tree: An Application of Inventory Account Auditing in Turkey
}

\author{
Alper Karavardar ${ }^{1}$ \\ ${ }^{1}$ Faculty of Economic and Administrative Sciences, Giresun University, Giresun, Turkey \\ Correspondence: Alper Karavardar, Faculty of Economic and Administrative Sciences, Giresun University, \\ Giresun, Turkey. Tel: 90-4543101300. E-mail: akaravardar@yahoo.com
}

Received: July 3, 2014

Accepted: July 23, 2014

Online Published: September 25, 2014

doi:10.5539/ijef.v6n10p214

URL: http://dx.doi.org/10.5539/ijef.v6n10p214

\begin{abstract}
Belief functions approach has a significant interest in audit literature. In this article, we present an evidential network for aggregating audit evidence based on belief function approach. This evidential network represents the structure of audit evidence for inventory account auditing. We used propagation of belief functions in an evidential network for aggregation of audit evidence. This approach help the auditors make an efficient and effective audit and it shows the relationship between audit evidence and audit risk. This study consists of three steps. In the first step, we reviewed the auditing literature which has relevant issues with this study. In the second step, we briefly gave information about Markov tree construction process and belief functions. In the third step, we created an evidential network which illustrates aggregating process for inventory account audit in a manufacturing company in Turkey. For this aim, we constructed a Markov tree. We used real audit files and we made use of auditors who are responsible in this case. We compared our evidential network results and auditors opinions which are represented in audit report. According to our study's result, our evidential network reflects auditors' professional judgement based on the audit evidence.
\end{abstract}

Keywords: belief functions, markov tree, auditing, Turkey

\section{Introduction}

Mathematical represantation of the relationship between the audit risk and the audit evidence is one of the most impontant topics in auditing literature. Audit risk model in the SAS 47(AICPA 1983) does not give a detailed explanation about the nature of this relationship. The proper determination of this relationship is an important issue to improve the audit quality and audit efficiency. Basically, audit risk is a result of combining the information which is provided by audit evidence. Audit evidence reflect the auditors' examinations which are relevant to management assertions on accounts of financial statements. An audit evidence may associate with more than one account, it may also associate with more than one management assertion. An audit risk illustrates the network structure that is based on the audit evidence and this network represents auditor's professional opinions. In other word, an audit risk is a result of an auditor's judgement process on management assertions in the light of audit procedures.

Using of Bayesian probability approach refers to a certainty about errors and misstatements' presence in an account. For example, if the auditor assigns a 0.25 value for inherent risk, under the Bayesian probability approach, it means the account is not materially misstated with 0.75 percent. Represantation of the auditor's ignorance which is related an issue based on Bayesian approach is different from the real world. Bayesian interpretation of audit risk model were subject of critism due to not fully reflecting the audit evidence uncertainty (Leslie, 1984; Akresh, Loebbecke \& Scott 1988; Srivastava \&Shafer, 1992). Belief function approach seems to be an outstanding discussion topic in auditing literature. Belief functions offer a flexible framework aggregating information from audit evidence and they reflect the uncertainty of audit evidence.

Belief functions approach has found many application opportunities in auditing (Srivastava, Mock, Pincus \& Wright 2012; Srivastava, Mock \& Gao 2011; Desai, Roberts and Srivastava 2010; Mock, Sun, Srivastava \& Vasarhelyi 2009; Srivastava \& Liu 2003). Srivastava (1995) studied propagation of belief functions which belongs to the audit evidence in network structure. Srivastava (1995) used Kong's (1986) algorithm which illustrates Markov tree construction to represent account receivables. Srivastava's (1995) study is important for represantation of relationship between audit risk and audit evidence because his study shows a propagation of 
belief. Thus, his study makes it possible to explain auditors' audit judgement.

There are limited studies in this area, our study aims to contribute to the literature. Besides, there are not any studies in a developing country. Thus, we chose Turkey as a study field. In this study, we took Srivastava's study (1995) as a theorical model. The Black Economy is a serious problem in Turkey, because it is a hidden part of the economy, where private cash transactions go unreported, and therefore untaxed. Thus, one of the significant audit issues in manufacturing companies is inventory account auditing in Turkey. For these reasons, we dealt with inventory account in our study. Legal authorities in Turkey, as Ministry of Finance, critise auditors judgements about this issue, thus we tried to explain Turkish auditors' professional judgements toward inventory account. We developed an evidential network which aggregates the audit evidence for inventory account auditing in a manufacturing company in Turkey. This study was conducted from December, 2010 to December, 2012. We examined whether the auditors achieved results with our evidential network's results which based on belief functions. The remainder of this paper is divided into four sections. In section one, we reviewed relevant auditing literature. In the second part of this study, we gave a brief information about construction of Markov tree and belief functions regarding to our aim. In the third part of this study, we created an evidential network with a Markov tree approach for inventory account audit and we aggregated whole information which are presented by audit evidence in the audit work file. We compared our results and auditors' professional opinions which were presented in the audit report. In the last section of this study, we discussed results and limitations.

\section{Literature Review}

Srivastava and Shafer (1992) discussed the structure of audit evidence that corresponds to a network of variables based on an audit risk model. According to them, the variables in the structure demostrate different audit aims, accounts and financial statement as a whole. Srivastava and Shafer (1992) argued the limitation of Bayesian audit risk interpretation and they offered belief function approach for the audit risk (plausibility of material misstatement) model. They showed propagation of belief functions ( $\mathrm{m}$ values) from the financial statement and accounts to the audit objectives in an 'and' tree. Their study is a significant concept for the auditing literature because they proposed an aggregation process for audit evidence pursuant to SAS 47 (1983). Their study have some limitations due to their simplifying assumptions. They considered only binary variables and they assumed each account or audit objective is equally important.

Srivastava, Shenoy and Srivastava (1995) described a basic method for propagating belief functions with 'and' tree in the financial audit. Srivastava (1997) asserted that using of probabilities to model an auditor's opinion about audit risks does not appropriate and he discussed the nature and the structure of the audit evidence. Srivastava and Liu (2003) discussed practices of belief functions in auditing and in business judgements. They proposed a generic evidential network for audit decision under the risk of fraud based on SAS 82. Shenoy and Shenoy (2002) used belief functions for modelling financial portfolios. Mock, Sun, Srivastava and Vasarhelyi (2009) argued belief functions for internal control risk assesment based on SOX. Srivastava, Mock and Turner (2009) comparatively illustrated two formulas for evaluating auditor's independence risk based on Bayesian approach and belief functions approach.

Desai, Roberts and Srivastava (2010), according to the section 302 of SOX requirement, proposed an analytical model for external auditor evaluation of the internal audit function that is based on belief function approach. Their internal audit function model was based on SAS 65 (AICPA, 1991) and PCAOB AS No.5 (PCAOB, 2007). Gao and Srivastava (2011) investigated the management fraud in financial statements and they separated fraud schemes: account schemes and evidence schemes. They used SEC allegations of fraud in Accounting and Auditing Enforcement Releases (AAERs) issued during the period 1997 and 2002. Srivastava, Mock and Gao (2011) used belief function appoach for fraud risk assesment. Fukakawa, Mock and Srivastava (2014) discussed assessing the risk of fraud at Olympus case in 1999 under the belief function approach. Their study have two levels: overall financial statement level and account level. They used Gao and Srivastava's findings in 2011. They used current standards for this case. All these studies show that there has been a substantial attention in the practice of belief function in the audit literature.

\section{Belief Functions And Markov Tree}

In this study we use belief function approach and Markov trees' properties. A Markov tree is an algorithm which is depicted by sets of nodes and edges. According to the definition of Markov tree, $N$ denotes nodes, $E$ denotes edges and $H$ denotes variables' subsets which based on belief functions. $(N, E)$ represents a tree and when $\mathrm{N}$ and

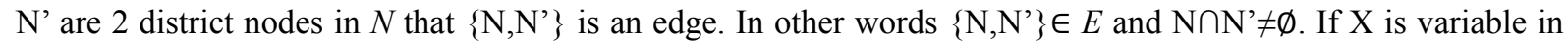
both N and N', X is in each node on the route from N to N'. Shenoy (1991) and Srivastava (1995) illustrate the construction process of Markov tree regarding pseudo-Pascal as given below: 


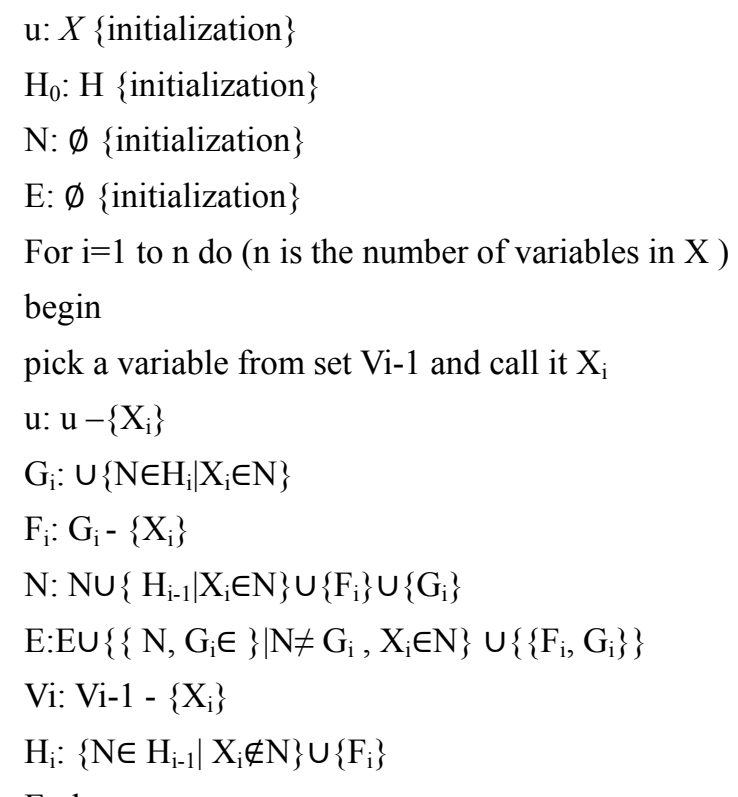

End.

In this algorithmic rule, each cycle delivers a Markov tree and in this study our aim is to construct a Markov tree. In this definition, it should have nodes in the tree as little as reachable. A Markov tree is an efficient way to propagate beliefs. We used Kong's (1986) “one-step-look-ahead” method to construct a Markov tree. In every iteration, Kong's method chooses a variable $X_{i}$ that is yield $G_{i}$ with the minimal number of variables.

The belief functions are based on mathematical theory of evidence 'Dempster Shafer theory of belief functions' (Shafer, 1976). This theory is based on the works of Arthur Dempster (1960) and Glenn Shafer (1976). This theory is especially relevant to both our study and the auditing as it focuses on evidence and evidential reasoning. Belief function, as distinct from Bayesian probability theory, can represent ignorance. While in the probability theory, ignorance situation is showed by assigning equal probabilities to the all possible outcomes, in the belief function theory ignorance situation is showed by a vacaous belief function. According to the belief functions approach, we use $\Theta$ notation for the representation of a frame (sample space) which an comprehensive and reciprocally specific set of feasible values of the variable. For example, $\Theta_{\mathrm{I}}=\{I, \sim \mathrm{I}\}$ is a frame with two elements in which ' $\mathrm{I}$ ' represents the inventory account balance that is not materially misstated and ' $\sim \mathrm{I}$ ' characterized as the inventory account balance that is materially misstated. According to the belief function approach, we can assign values (basic probability assignment-m values) to a subset of elements of frame. Basic probability assignment (bpa) is a function of ' $\mathrm{m}$ ' and ' $\mathrm{bpa}$ ' is representing the mathematical information in a belief function. The sum of all $\mathrm{m}$ values for all subsets of the frame is equal to 1 which alikes Bayesian approach. In auditing $\mathrm{m}$ values represent auditors' judgements based on audit evidence. For example, if the auditor believes there is no evidence to indicate the inventory account balance that is not fairly misstated and he/she assigned a 0.8 level of support based on who performed analytical procedures that implies remaining 0.2 level of support is uncommitted. We can represent this situation;

$$
\mathrm{m}(\mathrm{I})=0.8 \mathrm{~m}(\sim \mathrm{I})=0 \mathrm{~m}(\mathrm{I}, \sim \mathrm{I})=0.2[\mathrm{~m}(\varnothing)=0 \text { is represents the empty set }]
$$

Belief function term mathematically represents each subset of frame and auditor' beliefs in the subset. Belief in a subset $\mathrm{B}$ of a frame determines the total belief one has in based on the evidence represented through $\mathrm{m}$ values. It is defined as $\operatorname{Bel}(\mathrm{B})=\sum_{X \subseteq B} m(X)$ (Desai, Roberts and Srivastava, 2009). For instance according to example presented above

$$
\operatorname{Bel}[\mathrm{I}]=\mathrm{m}(\mathrm{I})=0.8, \operatorname{Bel}[\sim \mathrm{I}]=\mathrm{m}(\sim \mathrm{I})=0.2 \text { and } \operatorname{Bel}[\Theta]=\mathrm{m}(\mathrm{I})+\mathrm{m}(\sim \mathrm{I})+\mathrm{m}(\mathrm{I}, \sim \mathrm{I})=1.0
$$

According to the belief functions theory, a zero belief means there is no evidence to support the suggestion and there is lack of evidence. This property is different from Bayesian probability theory because in probability terms it means that the suggestion can't be true and it expresses impossibility. In the belief functions theory, plausibility function for Bel is defined by

$$
\operatorname{Pl}[\mathrm{I}]=1-\operatorname{Bel}(\sim \mathrm{I})
$$

In this notation the palusibility of $\mathrm{I}$ is a degree to which $\mathrm{I}$ is plausible based on evidence- the degree to which we do not misbelieve I or assign belief to its negation $\sim$ I (Srivastava, 1995). In our example, if the auditor completes 
ignorance about inventory account balance that it means Bel[I] $=0$ and $\mathrm{Pl}[\mathrm{I}]=1$. In the belief function appoach, the auditor can use Dempster's rule of combination for combining items of audit evidence which likes a Bayes' rule (Shafer, 1976). When it is considered two substantive items of evidence ( $m_{1}$ and $m_{2}$ for this frame), the combined $\mathrm{m}$ value for a subset $\mathrm{A}$ of frame using Dempster's combination rule is given by;

$$
\mathrm{m}(\mathrm{A})=\mathrm{K}^{-1} \sum\left\{\mathrm{m}_{1}\left(\mathrm{~B}_{1}\right) * \mathrm{~m}_{2}\left(\mathrm{~B}_{2}\right) \mid \mathrm{B}_{1} \cup \mathrm{B}_{2}=\mathrm{A}, \quad\right\} \text { where } \mathrm{K}=\sum\left\{\mathrm{m}_{1}\left(\mathrm{~B}_{1}\right) * \mathrm{~m}_{2}\left(\mathrm{~B}_{2}\right) \mid \mathrm{B}_{1} \cap \mathrm{B}_{2}=\right\}
$$

$\mathrm{K}$ shows the conflict between $\mathrm{m}_{1}$ and $\mathrm{m}_{2}$ (items of evidence). $\mathrm{K}=0$ means each item of evidence is contradict of other and in this situation we can not use Dempster's combination rule.

\section{An Application of Inventory Account Auditing}

In this part of the study, we illustrate using of belief functions approach for inventory account auditing. According to this aim, we made an evidential network which represents inventory account auditing. In this study, we use a real case data from a Turkish manufacturing company. We followed Srivastava's study (1995). In the first step, we examined audit files about inventory account balance. According to the audit files, auditors made 13 audit tests based on 4 audit procedures. In this case, the auditors team consists of 6 auditors: 1 senior manager, 1 supervisor, 2 seniors and 2 assistant staff. Their experience durations are 8 years, 5 years, 3 years and 1 year, respectively.

It is aimed here to make an evidential network in audit of inventory account to determine the association between the variables and the elements of audit evidence based on audit procedures. We created an evidential network. For representation of evidential network, we constructed a Markov Tree by using Kong's algorithm (1986). In auditing concept, an inventory account consists 5 managerial assertions: existence-occurence, completeness, rights and obligations, presentation and disclosure, valuation or allocation. It connotes that the inventory account balance is not materially misstated when the existence and properly valuation of audit objectives of the account have been fulfilled. In other words, if the auditors decide that the inventory exists and properly valued then they accept the inventory account presented fairly. Figure 1. demonstrates the evidential network for the audit of inventory account. Each audit test in audit field is a confirmation intended one or more spesific management assertions. For example, if the auditors make a test for monitoring the client's physical inventory, this audit evidence gives some information about existence and rights assertions. We showed related tests and related management assertions in Figure 1. We used two important propagation processes that are vacaous extension and marginalization. If $\mathrm{m}$ values are forwarded from a smaller node to a bigger node then we used vacaous extension. If $\mathrm{m}$ values are forwarded from bigger node to a smaller node then we used marginalization concept. In this study, we represented eight variables: I, E,V, EO, CP, RO, PD, VA. We used 'AND' relationship between these variables. An 'AND' relationship intimates that the variable on its left is met if only the variables on its right are met (Srivastava, 1995). We used a circle with ' $\&$ ' notation in it to represent an 'AND' node with same principle. Our general notation is similiar Srivastava's notation. We used capital letter(s) to symbolize variable's name (such as 'I' represent Inventory) and small letter(s) to symbolize its values.

Table 1 represents a construction of Markov tree steps according to the Kong's one-step-look-ahead algorithm Each step represents an iteration for relevant variables. As a result of this algorithm, we created the Markov tree in Figure 2. Figure 2. is an illustration of audit evidence relations. Propagation of $\mathrm{m}$ values (belief functions) in the Markov tree in Figure 2. was showed in table 2, 3, 4, 5, 6 and 7. For example we showed aggregration of belief functions for existence and rights assertions in Table 7. We wanted the auditors to assign $\mathrm{m}$ values for audit evidence. We used their $\mathrm{m}$ values and we applied these figures to our evidential network. Basically $\mathrm{m}$ values represent level of supports of audit evidence based on audit procedures. Each audit procedure consists of different tests and each test result represents different $m$ values subsequent management assertions.

According to the Figure 1., after aggregating all the audit evidence in audit files the whole $\mathrm{m}$ values at node 1 is provided by ( see the end of Table 7):

$$
\mathrm{m}_{17}^{\mathrm{t}}(\mathrm{I})=0.79 \mathrm{~m}_{17}^{\mathrm{t}}(\sim \mathrm{I})=0 \mathrm{~m}_{17}^{\mathrm{t}}(\{\mathrm{I}, \sim \mathrm{I}\})=\mathrm{m}_{17}^{\mathrm{t}}\left(\Theta_{17}\right)=0.21
$$

The corresponding belief and plausibilites are asserted by (see Srivastava and Shafer 1992 for explanations):

$$
\operatorname{Bel}_{\mathrm{I}}^{\mathrm{t}}(\mathrm{I})=0.79 \operatorname{Bel}_{\mathrm{I}}^{\mathrm{t}}(\sim \mathrm{I})=0 \operatorname{Bel}_{\mathrm{I}}^{\mathrm{t}}(\{\mathrm{I}, \sim \mathrm{I}\})=1
$$

and

$$
\mathrm{Pl}_{\mathrm{I}}^{\mathrm{t}}(\mathrm{I})=1-\operatorname{Bel}_{\mathrm{I}}^{\mathrm{t}}(\sim \mathrm{I})=1 \quad \mathrm{Pl}_{\mathrm{I}}^{\mathrm{t}}(\sim \mathrm{I})=1-\operatorname{Bel}_{\mathrm{I}}^{\mathrm{t}}(\mathrm{I})=0.21
$$

These outcomes point out that the total belief 0.79 that the inventory account balance is not materially misstated and a plausibilty 0.21 that account materially misstated. According to Srivastava and Shafer (1992), we obtained 0.21 risk that the inventory account materially misstated. We reviewed audit report about inventory account. 
According to the audit report there is no important problems in invertory account and it is fairly represented based on US and TR GAAS. In other words, our evidential network can reflect auditors' opinions in this case.

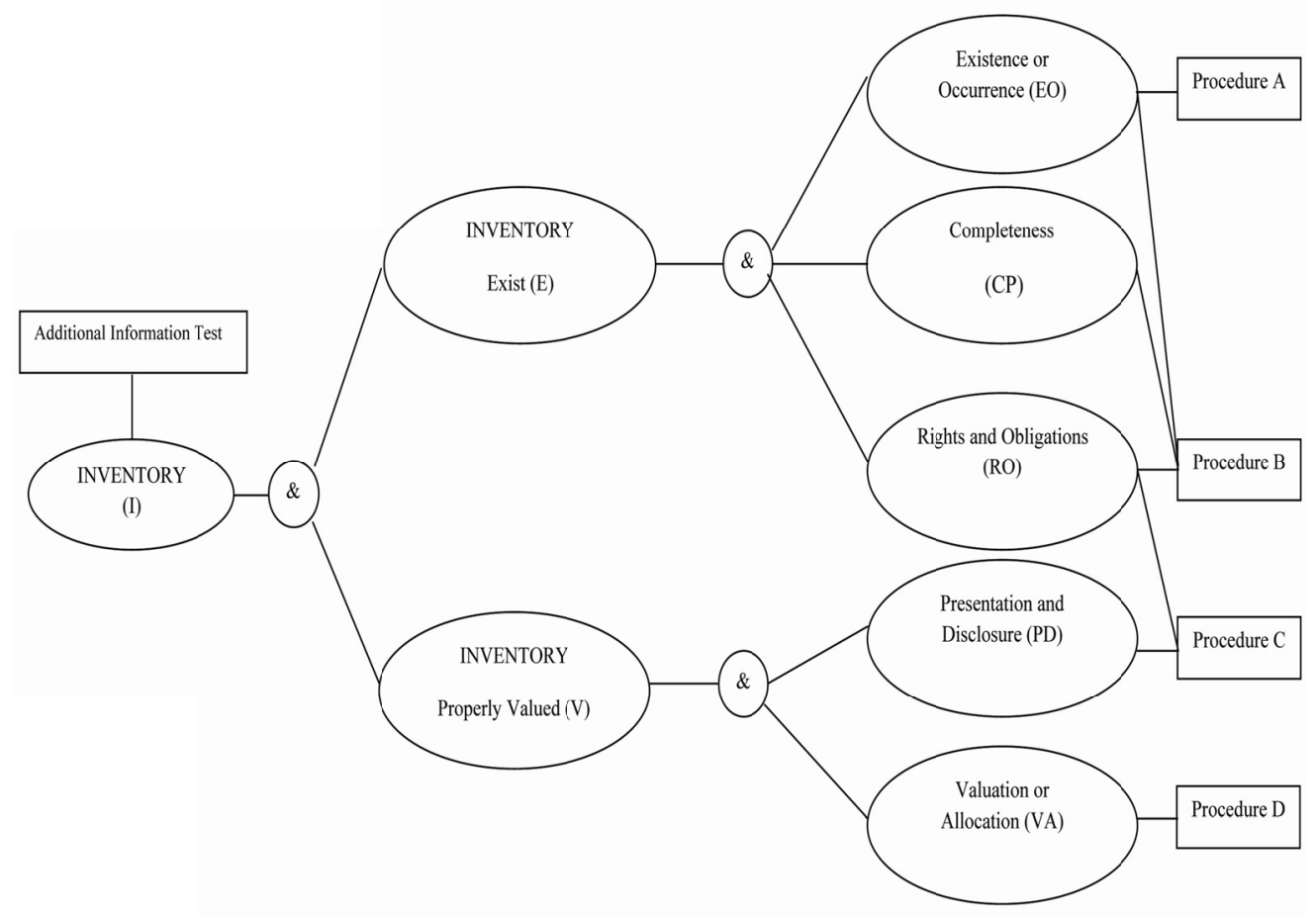

Figure 1. Evidential network for inventory account auditing

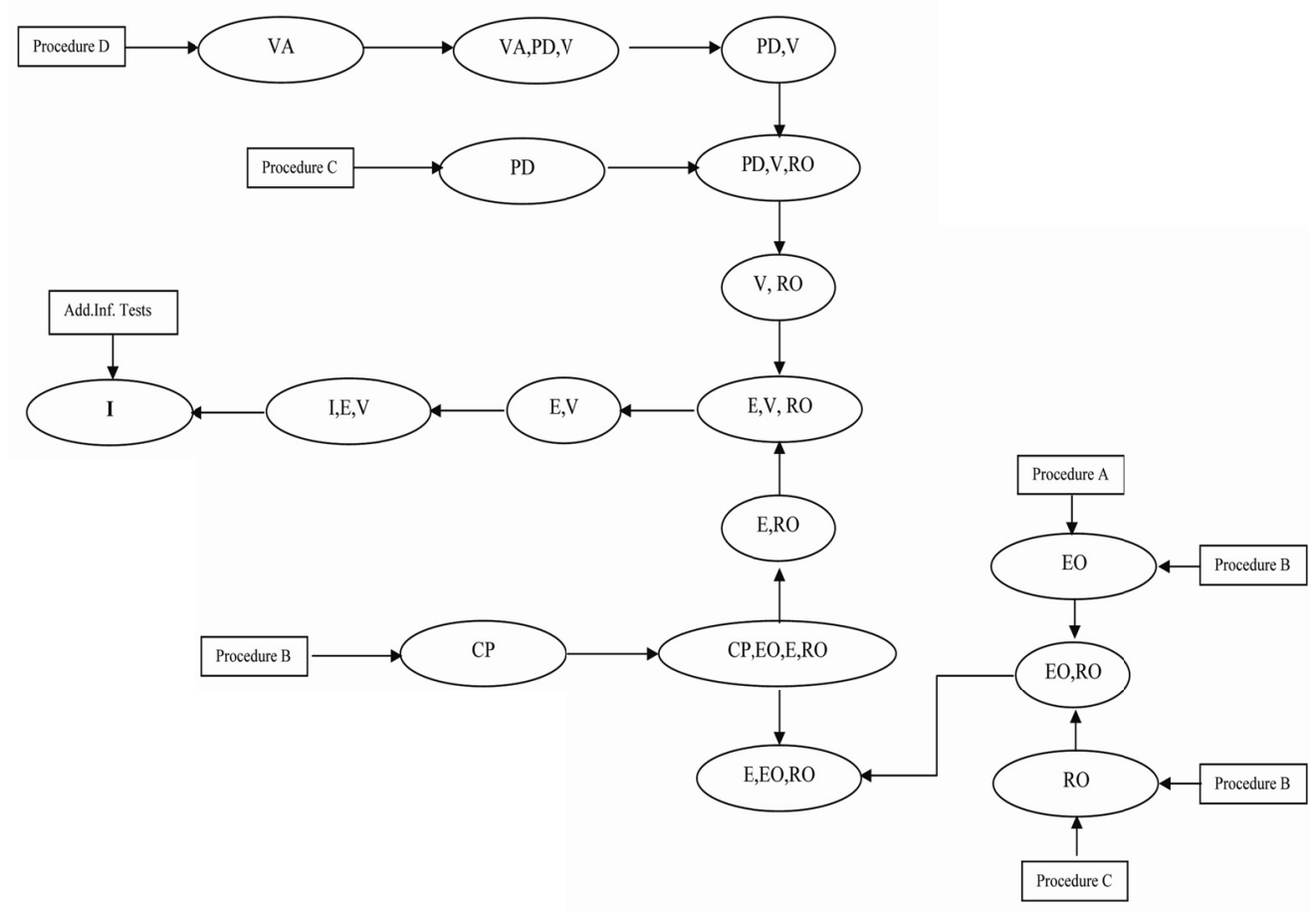

Figure 2. Markov tree of the evidential network in figure 1 for inventory account auditing 
Table 1. Markov tree construction process for the figure 1

\begin{tabular}{|c|c|c|c|}
\hline \multicolumn{4}{|c|}{$\begin{array}{l}\boldsymbol{X}=\{\mathbf{I}, \mathbf{E}, \mathbf{V}, \mathbf{E O}, \mathbf{C P}, \mathbf{R O}, \mathbf{P D}, \mathbf{V A}\} \\
\boldsymbol{H}=\{\{\mathbf{I}\},\{\mathbf{E}, \mathbf{V}\},\{\mathbf{E O}\},\{\mathbf{C P}\},\{\mathbf{R O}\},\{\mathbf{P D}\},\{\mathbf{V} \\
\text { Initialization: } \boldsymbol{V}_{\boldsymbol{0}}=X, H_{0}=H, N=\emptyset, E=\emptyset\end{array}$} \\
\hline $\begin{array}{l}\text { Iteration } \\
\text { Number } \\
\text { i }\end{array}$ & $\begin{array}{c}\mathbf{X}_{\mathrm{i}} \text { and } \mathrm{G}_{\mathrm{i}}, \text { where } \\
\mathbf{G}_{\mathbf{i}}=\mathrm{U}\left\{\mathbf{N} \in \boldsymbol{H}_{\boldsymbol{i}-1} \mid \mathbf{X}_{\mathrm{i}} \in \mathbf{N}\right\}\end{array}$ & $\begin{array}{c}\text { Number of } \\
\text { elements in } G_{i}\end{array}$ & $\begin{array}{c}\mathrm{X}_{\mathrm{i}}, \mathrm{G}_{\mathrm{i}}, \mathrm{F}_{\mathrm{i}}, N, E, V_{i}, H_{i} \text { with the minimal number of } \\
\text { elements in } \mathrm{G}_{\mathrm{i}} \text { employing the algorithmic rule in Section } \\
\qquad 3\end{array}$ \\
\hline 1 & $\begin{array}{l}\text { CP: }\{C P, E O, R O, E\} \\
\text { E: }\{I, E, V, E O, C P, R O, P D\} \\
\text { EO: }\{E O, C P, R O, E\} \\
\text { I: }\{I, E, V\} \\
\text { PD: }\{P D, V A, V, R O\} \\
\text { RO: }\{R O, C P, E O, E, P D\} \\
\text { V: }\{I, E, V, P D, V A, R O\} \\
\text { VA: }\{V A, P D, V\}\end{array}$ & $\begin{array}{l}4 \\
7 \\
4 \\
3 * \\
4 \\
5 \\
6 \\
3\end{array}$ & $\begin{array}{l}\mathrm{X}_{1}: \mathrm{I}, \mathrm{G}_{1}=\{\mathrm{I}, \mathrm{E}, \mathrm{V}\}, \mathrm{F}_{1}=\mathrm{G}_{1}-\mathrm{X}_{1}=\{\mathrm{E}, \mathrm{V}\} \\
N=\left\{\{\mathrm{I}\}, \mathrm{G}_{1}, \mathrm{~F}_{1}\right\} \\
\left.E=\left\{\{\mathrm{I}\}, \mathrm{G}_{1}, \mathrm{~F}_{1}\right\},\left\{\mathrm{G}_{1}, \mathrm{~F}_{1}\right\}\right\} \\
V_{l}=V_{0}-\mathrm{X}_{1}=\{\mathrm{E}, \mathrm{V}, \mathrm{EO}, \mathrm{CP}, \mathrm{RO}, \mathrm{PD}, \mathrm{VA}\} \\
H_{1}=\{\{\mathrm{E}, \mathrm{V}\},\{\mathrm{EO}\},\{\mathrm{CP}\},\{\mathrm{RO}\},\{\mathrm{PD}\},\{\mathrm{VA}\},\{\mathrm{PD}, \mathrm{RO}\} \\
\{\mathrm{V}, \mathrm{VA}, \mathrm{PD}\},\{\mathrm{E}, \mathrm{EO}, \mathrm{CP}, \mathrm{RO}\}\}\end{array}$ \\
\hline 2 & $\begin{array}{l}\text { CP: }\{C P, E O, R O, E\} \\
\text { E: }\{E, V, E O, C P, R O, P D\} \\
\text { EO: }\{E O, C P, R O, E\} \\
\text { PD: }\{P D, V A, V, R O\} \\
\text { RO: }\{R O, C P, E O, E, P D\} \\
V:\{E, V, P D, V A, R O\} \\
V A:\{V A, P D, V\}\end{array}$ & $\begin{array}{l}4 \\
6 \\
4 \\
4 \\
5 \\
5 \\
3 *\end{array}$ & $\begin{array}{l}\mathrm{X}_{2}: \mathrm{VA}, \mathrm{G}_{2}=\{\mathrm{VA}, \mathrm{PD}, \mathrm{V}\}, \mathrm{F}_{2}=\mathrm{G}_{2}-\mathrm{X}_{2}=\{\mathrm{PD}, \mathrm{V}\}, \\
\text { These new nodes to be supplemented to } N \text { are: }\{\mathrm{VA}\}, \mathrm{G}_{2}, \\
\mathrm{~F}_{2} \\
\text { These new edges to be supplemented to } E \text { are: }\{\{\mathrm{VA}\}, \\
\left.\left.\mathrm{G}_{2}\right\},\left\{\mathrm{G}_{2}, \mathrm{~F}_{2}\right\}\right\} \\
V_{2}=V_{1}-\mathrm{X}_{2}=\{\mathrm{E}, \mathrm{V}, \mathrm{EO}, \mathrm{CP}, \mathrm{RO}, \mathrm{PD}\} \\
H_{2}=\{\{\mathrm{E}, \mathrm{V}\},\{\mathrm{EO}\},\{\mathrm{CP}\},\{\mathrm{RO}\},\{\mathrm{PD}\},\{\mathrm{PD}, \mathrm{RO}\},\{\mathrm{V}, \\
\mathrm{PD}\},\{\mathrm{E}, \mathrm{EO}, \mathrm{CP}, \mathrm{RO}\}\}\end{array}$ \\
\hline 3 & $\begin{array}{l}\mathrm{CP}:\{\mathrm{CP}, \mathrm{EO}, \mathrm{RO}, \mathrm{E}\} \\
\mathrm{E}:\{\mathrm{E}, \mathrm{V}, \mathrm{EO}, \mathrm{CP}, \mathrm{RO}, \mathrm{PD}\} \\
\mathrm{EO}:\{\mathrm{EO}, \mathrm{CP}, \mathrm{RO}, \mathrm{E}\} \\
\mathrm{PD}:\{\mathrm{PD}, \mathrm{V}, \mathrm{RO}\} \\
\mathrm{RO}:\{\mathrm{RO}, \mathrm{CP}, \mathrm{EO}, \mathrm{E}, \mathrm{PD}\} \\
\mathrm{V}:\{\mathrm{E}, \mathrm{V}, \mathrm{PD}, \mathrm{RO}\}\end{array}$ & $\begin{array}{l}4 \\
6 \\
4 \\
3 * \\
5 \\
4\end{array}$ & $\begin{array}{l}\mathrm{X}_{3}: \mathrm{PD}, \mathrm{G}_{3}=\{\mathrm{PD}, \mathrm{V}, \mathrm{RO}\}, \mathrm{F}_{3}=\mathrm{G}_{3}-\mathrm{X}_{3}=\{\mathrm{RO}, \mathrm{V}\} \\
\text { These new nodes to be supplemented to } N \text { are: }\{\mathrm{PD}\}, \mathrm{G}_{3}, \\
\mathrm{~F}_{3} \\
\text { These new edges to be supplemented to } E \text { are: }\{\{\mathrm{PD}\} \\
\left.\left.\mathrm{G}_{3}\right\},\left\{\mathrm{G}_{3}, \mathrm{~F}_{3}\right\}\right\} \\
V_{3}=V_{2}-\mathrm{X}_{3}=\{\mathrm{E}, \mathrm{V}, \mathrm{EO}, \mathrm{CP}, \mathrm{RO}\} \\
H_{3}=\{\{\mathrm{E}, \mathrm{V}\},\{\mathrm{EO}\},\{\mathrm{CP}\},\{\mathrm{RO}\},\{\mathrm{RO}\},\{\mathrm{V}\},\{\mathrm{E}, \mathrm{EO}, \mathrm{CP}, \\
\mathrm{RO}\}\}\end{array}$ \\
\hline 4 & $\begin{array}{l}\text { CP: }\{\mathrm{CP}, \mathrm{EO}, \mathrm{RO}, \mathrm{E}\} \\
\mathrm{E}:\{\mathrm{E}, \mathrm{V}, \mathrm{EO}, \mathrm{CP}, \mathrm{RO}\} \\
\mathrm{EO}:\{\mathrm{EO}, \mathrm{CP}, \mathrm{RO}, \mathrm{E}\} \\
\mathrm{RO}:\{\mathrm{RO}, \mathrm{CP}, \mathrm{EO}, \mathrm{E}\} \\
\mathrm{V}:\{\mathrm{E}, \mathrm{V}, \mathrm{RO}\}\end{array}$ & $\begin{array}{l}4 \\
5 \\
4 \\
4 \\
3 *\end{array}$ & $\begin{array}{l}\mathrm{X}_{4}: \mathrm{V}, \mathrm{G}_{4}=\{\mathrm{E}, \mathrm{V}, \mathrm{RO}\}, \mathrm{F}_{4}=\mathrm{G}_{4}-\mathrm{X}_{4}=\{\mathrm{E}, \mathrm{RO}\}, \\
\text { These new nodes to be supplemented to } N \text { are: }\{\mathrm{V}\}, \mathrm{G}_{4}, \mathrm{~F}_{4} \\
\text { These new edges to be supplemented to } E \text { are: }\{\{\mathrm{V}\} \\
\left.\left.\mathrm{G}_{4}\right\},\left\{\mathrm{G}_{4}, \mathrm{~F}_{4}\right\}\right\} \\
V_{4}=V_{3}-\mathrm{X}_{4}=\{\mathrm{E}, \mathrm{EO}, \mathrm{CP}, \mathrm{RO}\} \\
H_{4}=\{\{\mathrm{E}\},\{\mathrm{EO}\},\{\mathrm{CP}\},\{\mathrm{RO}\},\{\mathrm{RO}\},\{\mathrm{E}, \mathrm{EO}, \mathrm{CP}, \mathrm{RO}\}\}\end{array}$ \\
\hline
\end{tabular}

Table 1. Markov tree construction process for the figure 1 (Continued)

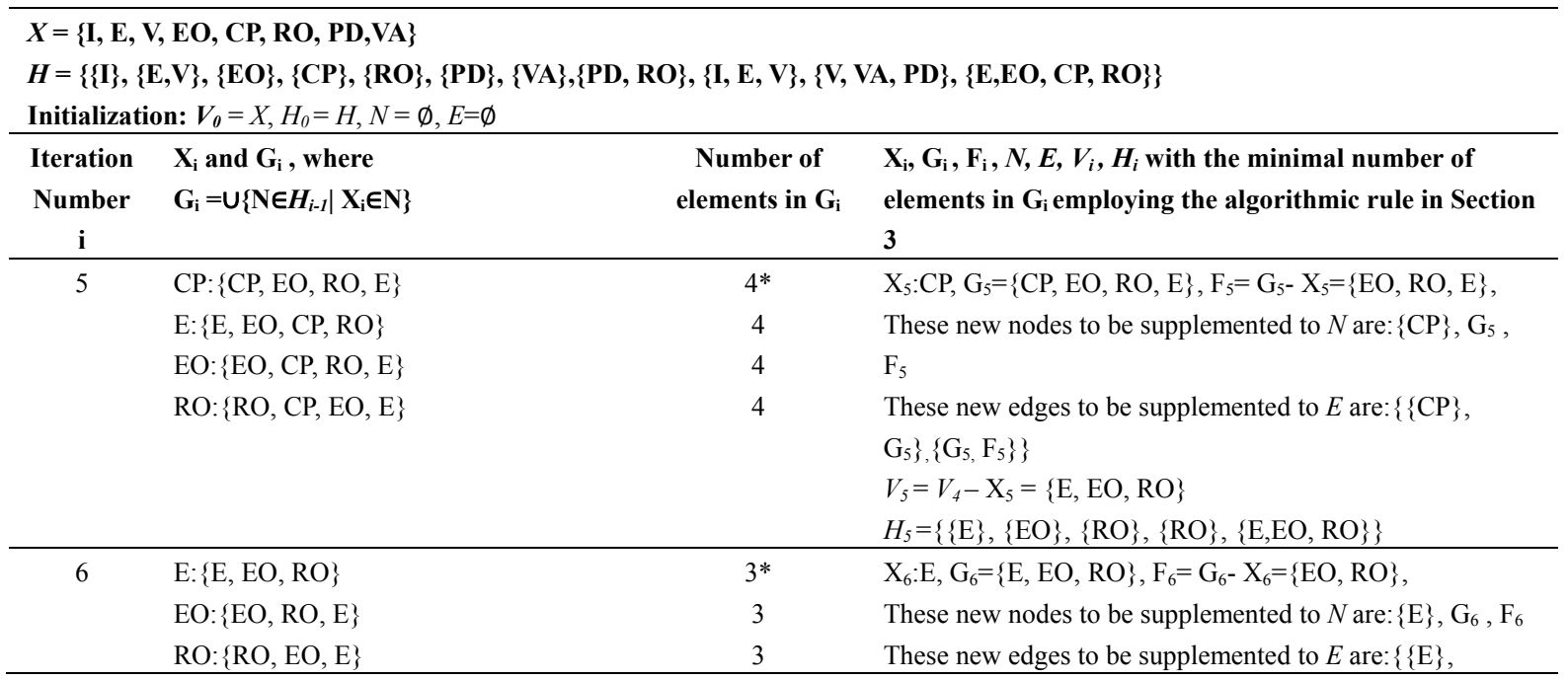




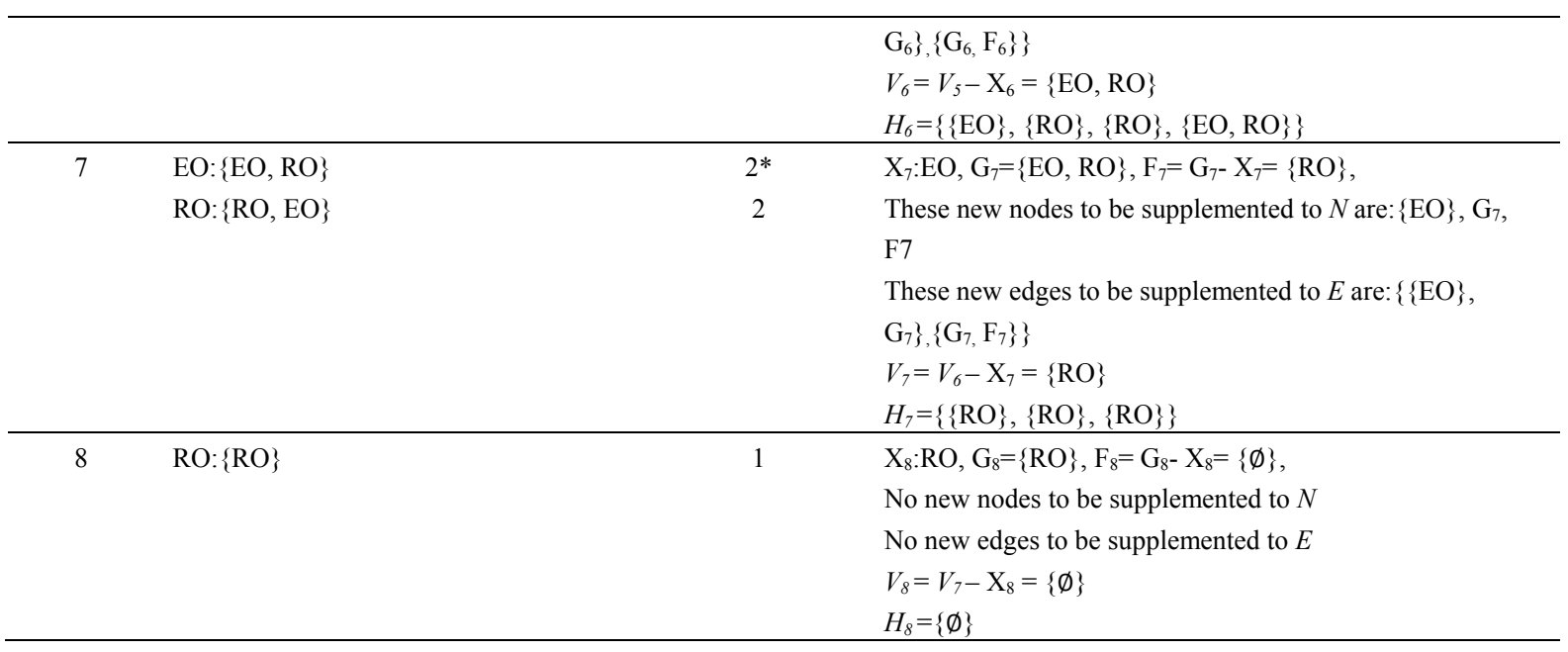

Table 2. Propagation of $m$ values (belief functions) in the Markov tree in Figure 2. for node 1, 2, 3 and 4

\begin{tabular}{|c|c|c|c|c|}
\hline Node's No & The frame of the node & $\begin{array}{l}\text { m-values identified } \\
\text { at the node }\end{array}$ & $\begin{array}{l}\text { m-values at the node from } \\
\text { contiguous node/nodes }\end{array}$ & $\begin{array}{c}\text { the sum of m-values at the node } \\
\text { (received from compounding } \\
\text { m-values in colums } 3 \text { and } 4 \\
\text { using Dempster's combination } \\
\text { rule) }\end{array}$ \\
\hline 1. & $\Theta_{1}=\{\mathrm{VA}, \sim \mathrm{VA}\}$ & $\begin{array}{l}\mathrm{m}_{1 \text { Test } 5}(\mathrm{VA})=0.2 \\
\mathrm{~m}_{1 \text { Test } 5}\left(\Theta_{1}\right)=0.8 \\
\mathrm{~m}_{1 \text { Test } 6}(\mathrm{VA})=0.3 \\
\mathrm{~m}_{1 \text { Test } 6}\left(\Theta_{1}\right)=0.7 \\
\mathrm{~m}_{1 \text { Test } 7}(\mathrm{VA})=0.2 \\
\mathrm{~m}_{1 \text { Test } 7}\left(\Theta_{1}\right)=0.8 \\
\mathrm{~m}_{1 \text { Test } 8}(\mathrm{VA})=0.4 \\
\mathrm{~m}_{1 \text { Test } 8}\left(\Theta_{1}\right)=0.6\end{array}$ & none & $\begin{array}{l}\mathrm{m}_{1}^{\mathrm{t}}(\mathrm{VA})=0.73 \\
\mathrm{~m}_{1}^{\mathrm{t}}\left(\Theta_{1}\right)=0.27\end{array}$ \\
\hline 2. & $\begin{array}{l}\Theta_{1}^{\#}=\{(\mathrm{V}, \mathrm{VA}, \mathrm{PD}) \\
(\sim \mathrm{V}, \mathrm{VA}, \sim \mathrm{PD}) \\
(\sim \mathrm{V}, \sim \mathrm{VA}, \mathrm{PD}) \\
(\sim \mathrm{V}, \sim \mathrm{VA}, \sim \mathrm{PD})\}\end{array}$ & $\mathrm{m} 2(\Theta 2)=1.0$ & $\begin{array}{l}\mathrm{m}_{1} \oplus_{2}(\{(\mathrm{~V}, \mathrm{VA}, \mathrm{PD}),(\sim \mathrm{V}, \mathrm{VA}, \sim \mathrm{PD})\}= \\
\mathrm{m}_{2}^{\mathrm{t}}(\mathrm{VA})=0.73 \\
\mathrm{~m}_{1 \phi_{2}}\left(\Theta_{2}\right)=\mathrm{m}_{1}^{\mathrm{t}}\left(\Theta_{1}\right)=0.27\end{array}$ & $\begin{array}{l}\mathrm{m}_{2}^{\mathrm{t}}(\{(\mathrm{V}, \mathrm{VA}, \mathrm{PD}),(\sim \mathrm{V}, \mathrm{VA}, \sim \mathrm{PD})\})= \\
0.73 \\
\mathrm{~m}_{2}^{\mathrm{t}}\left(\Theta_{2}\right)=0.27\end{array}$ \\
\hline 3. & $\begin{array}{l}\Theta_{3}=\{(\mathrm{V}, \mathrm{PD}),(\mathrm{V}, \sim \mathrm{PD}), \\
(\sim \mathrm{V}, \mathrm{PD}) \\
(\sim \mathrm{V}, \sim \mathrm{PD})\}\end{array}$ & $\mathrm{m}_{3}\left(\Theta_{3}\right)=1.0$ & 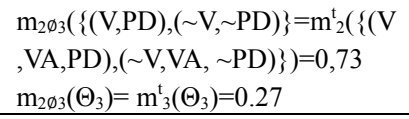 & $\begin{array}{l}\mathrm{m}_{3}^{\mathrm{t}}(\{(\mathrm{V}, \mathrm{PD}),(\sim \mathrm{V}, \sim \mathrm{PD})\})=0.73 \\
\mathrm{~m}_{3}^{\mathrm{t}}\left(\Theta_{3}\right)=0.27\end{array}$ \\
\hline 4. & $\Theta_{5}=\{\mathrm{PD}, \sim \mathrm{PD}\}$ & $\begin{array}{l}\mathrm{m}_{4 \text { Test } 10}(\mathrm{PD})=0.15 \\
\mathrm{~m}_{4 \text { Test } 10}\left(\Theta_{4}\right)=0.85 \\
\mathrm{~m}_{4 \text { Test11 }}(\mathrm{PD})=0.2 \\
\mathrm{~m}_{4 \text { Test11 }}\left(\Theta_{4}\right)=0.8 \\
\mathrm{~m}_{4 \text { Test12 }}(\mathrm{PD})=0.3 \\
\mathrm{~m}_{4 \text { Test } 13}\left(\Theta_{4}\right)=0.7\end{array}$ & none & $\begin{array}{l}\mathrm{m}_{4}^{\mathrm{t}}(\mathrm{PD})=0.52 \\
\mathrm{~m}_{4}^{\mathrm{t}}\left(\Theta_{4}\right)=0.48\end{array}$ \\
\hline
\end{tabular}

Table 3. Propagation of $m$ values (belief functions) in the Markov tree in figure 2 for node 5, 6 and 7

\begin{tabular}{|c|c|c|c|c|}
\hline Node & $\begin{array}{l}\text { Frame of the } \\
\text { node }\end{array}$ & $\begin{array}{c}\text { m-values } \\
\text { defined at the } \\
\text { node }\end{array}$ & $\begin{array}{l}\text { m-values at the node from neighboring } \\
\text { node/nodes }\end{array}$ & $\begin{array}{l}\text { the total m-values at the node } \\
\text { (obtained from combining } \\
\text { m-values in colums } 3 \text { and } 4 \text { using } \\
\text { Dempster's combination rule) }\end{array}$ \\
\hline 5. & $\begin{array}{l}\Theta_{5}^{\# \#}=\{(\mathrm{V}, \mathrm{PD}, \mathrm{RO} \\
), \quad(\sim \mathrm{V}, \sim \mathrm{PD}, \mathrm{RO}) \\
(\sim \mathrm{V}, \mathrm{PD}, \sim \mathrm{RO}) \\
(\sim \mathrm{V}, \sim \mathrm{PD}, \sim \mathrm{RO})\}\end{array}$ & $\mathrm{m}_{5}\left(\Theta_{5}\right)=1.0$ & $\begin{array}{l}\mathrm{m}_{4 \emptyset_{5}}(\{(\mathrm{~V}, \mathrm{PD}, \mathrm{RO}),(\sim \mathrm{V}, \mathrm{PD}, \sim \mathrm{RO})\})=\mathrm{m}_{4}^{\mathrm{t}}(\mathrm{PD})=0.52 \\
\mathrm{~m}_{4 \emptyset_{5}}\left(\Theta_{5}\right)=\mathrm{m}_{4}^{\mathrm{t}}\left(\Theta_{4}\right)=0.48 \\
\mathrm{~m}_{3 \emptyset_{5}}=(\{(\mathrm{V}, \mathrm{PD}, \mathrm{RO}),(\sim \mathrm{V}, \sim \mathrm{PD}, \mathrm{RO}),(\sim \mathrm{V}, \sim \mathrm{PD}, \sim \mathrm{RO})\} \\
=\mathrm{m}_{3}^{\mathrm{t}}(\{(\mathrm{V}, \mathrm{PD}),(\sim \mathrm{V}, \sim \mathrm{PD})\})=0.73\end{array}$ & $\begin{array}{l}\mathrm{m}_{5}^{\mathrm{t}}(\mathrm{V}, \mathrm{PD}, \mathrm{RO})=(0.52 * 0.73)=0.3796 \\
\mathrm{~m}_{5}^{\mathrm{t}}(\{(\mathrm{V}, \mathrm{PD}, \mathrm{RO}),(\sim \mathrm{V}, \sim \mathrm{PD}, \mathrm{RO})\}) \\
=(0.52 * 0.27)=0.1404 \\
\mathrm{~m}_{5}^{\mathrm{t}}(\{(\mathrm{V}, \mathrm{PD}, \mathrm{RO}),(\sim \mathrm{V}, \mathrm{PD}, \sim \mathrm{RO})\})\end{array}$ \\
\hline
\end{tabular}




\begin{tabular}{|c|c|c|c|c|}
\hline & & & $\mathrm{m}_{2} \emptyset_{3}\left(\Theta_{3}\right)=\mathrm{m}_{3}^{\mathrm{t}}\left(\Theta_{3}\right)=0.12 \mathrm{~m}_{3} \emptyset_{5}\left(\Theta_{5}\right)=\mathrm{m}_{3}^{\mathrm{t}}\left(\Theta_{3}\right)=0.27$ & $\begin{array}{l}=(0.48 * 0.73)=0.3504 \\
\mathrm{~m}_{5}^{\mathrm{t}}\left(\Theta_{5}\right)=(0.48 * 0.27)=0.1296\end{array}$ \\
\hline 6. & $\begin{array}{l}\Theta_{6}=\{(\mathrm{V}, \mathrm{RO}) \\
(\mathrm{V}, \sim \mathrm{RO}) \\
(\sim \mathrm{V}, \mathrm{RO}) \\
(\sim \mathrm{V}, \sim \mathrm{RO})\}\end{array}$ & $\mathrm{m}_{6}\left(\Theta_{6}\right)=1.0$ & $\begin{array}{l}\mathrm{m}_{5 \emptyset_{6}}(\mathrm{~V}, \mathrm{RO})=\mathrm{m}_{5}^{\mathrm{t}}(\mathrm{V}, \mathrm{PD}, \mathrm{RO})=0.3796 \\
\mathrm{~m}_{5 \emptyset_{6}}(\sim \mathrm{V}, \mathrm{RO})=\mathrm{m}_{5}^{\mathrm{t}}(\{(\mathrm{V}, \mathrm{PD}, \mathrm{RO}),(\sim \mathrm{V}, \sim \mathrm{PD}, \mathrm{RO})\}) \\
=0.1404 \\
\mathrm{~m}_{5 \emptyset_{6}}(\sim \mathrm{V}, \sim \mathrm{RO})=\mathrm{m}_{5}^{\mathrm{t}}(\{(\mathrm{V}, \mathrm{PD}, \mathrm{RO}),(\sim \mathrm{V}, \mathrm{PD}, \sim \mathrm{RO})\}) \\
=0.3504 \\
\mathrm{~m}_{5 \emptyset_{6}}\left(\Theta_{6}\right)=\mathrm{m}_{5}^{\mathrm{t}}\left(\Theta_{5}\right)=0.1296\end{array}$ & $\begin{array}{l}\mathrm{m}_{6}^{\mathrm{t}}(\mathrm{V}, \mathrm{RO})=0.3796 \\
\mathrm{~m}_{6}^{\mathrm{t}}(\{(\mathrm{V}, \mathrm{RO}),(\sim \mathrm{V}, \mathrm{RO})\})=0.1404 \\
\mathrm{~m}_{6}^{\mathrm{t}}(\{(\mathrm{V}, \mathrm{RO}),(\sim \mathrm{V}, \sim \mathrm{RO})\})=0.3504 \\
\mathrm{~m}_{3}^{\mathrm{t}}\left(\Theta_{3}\right)=0.1296\end{array}$ \\
\hline 7. & $\Theta_{7}=\{\mathrm{EO}, \sim \mathrm{EO}\}$ & $\begin{array}{l}\mathrm{m}_{\text {7Test3 }}(\mathrm{EO})=0.4 \\
\mathrm{~m}_{\text {7Test3 }}\left(\Theta_{7}\right)=0.6 \\
\mathrm{~m}_{1 \text { Test6 }}(\mathrm{VA})=0.2 \\
\mathrm{~m}_{1 \text { Test6 }}\left(\Theta_{1}\right)=0.8 \\
\mathrm{~m}_{1 \text { Test7 }}(\mathrm{VA})=0.3 \\
\mathrm{~m}_{1 \text { Test }}\left(\Theta_{1}\right)=0.7 \\
\mathrm{~m}_{1 \text { Test } 8}(\mathrm{VA})=0.1 \\
\mathrm{~m}_{1 \text { Test } 8}\left(\Theta_{1}\right)=0.9\end{array}$ & none & $\begin{array}{l}\mathrm{m}_{7}^{\mathrm{t}}(\mathrm{EO})=0.7 \\
\mathrm{~m}_{7}^{\mathrm{t}}\left(\Theta_{7}\right)=0.3\end{array}$ \\
\hline$(\sim \mathrm{V}$ & $\begin{array}{l}\text { al this node } \\
\text { )) \}. But acco } \\
\text { ressponding } n\end{array}$ & $\begin{array}{l}\text { is } 8 \text { elements: } \\
\text { o "AND" relati } \\
\text { elements are }\{0\end{array}$ & $\begin{array}{l}\text { JPD,RO),(V,PD, RO),(V, PD,RO),( V,PD,RO), } \\
\text { hip (V,PD, } \sim \mathrm{RO}),(\mathrm{V}, \sim \mathrm{PD}, \mathrm{RO}),(\sim \mathrm{V}, \mathrm{PD}, \mathrm{RO}),(\mathrm{V}, \sim \mathrm{PI} \\
\mathrm{D}, \mathrm{RO}),(\sim \mathrm{V}, \sim \mathrm{PD}, \mathrm{RO}),(\sim \mathrm{V}, \mathrm{PD}, \sim \mathrm{RO}),(\sim \mathrm{V}, \sim \mathrm{PD}, \sim \mathrm{H}\end{array}$ & $\begin{array}{l}, \sim \mathrm{RO}),(\sim \mathrm{V}, \sim \mathrm{PD}, \mathrm{RO}),(\sim \mathrm{V}, \mathrm{PD}, \sim \mathrm{RO} \\
\text { ) elements are impossible. For th}\end{array}$ \\
\hline
\end{tabular}

Table 4. Propagation of $m$ values (belief functions) in the Markov tree in Figure 2. for node 8, 9 and 10

\begin{tabular}{|c|c|c|c|c|}
\hline Node & Frame of the node & m-values defined at the node & $\begin{array}{l}\text { m-values at the node from } \\
\text { neighboring node/nodes }\end{array}$ & $\begin{array}{l}\text { the total m-values at the node } \\
\text { (obtained from combining } \\
\text { m-values in colums } 3 \text { and } 4 \\
\text { using Dempster's } \\
\text { combination rule) }\end{array}$ \\
\hline 8. & $\Theta_{8}=\{\mathrm{RO}, \sim \mathrm{RO}\}$ & $\begin{array}{l}\mathrm{m}_{8 \text { Test } 10}(\mathrm{RO})=0.1 \mathrm{~m}_{8 \mathrm{Test} 10}\left(\Theta_{8}\right)=0.9 \\
\mathrm{~m}_{8 \text { Test } 11}(\mathrm{RO})=0.3 \quad \mathrm{~m}_{8 \mathrm{Tes} 11}\left(\Theta_{8}\right)=0.7 \\
\mathrm{~m}_{8 \text { Test } 12}(\mathrm{RO})=0.2 \mathrm{~m}_{8 \mathrm{Tes} 12}\left(\Theta_{8}\right)=0.8 \\
\mathrm{~m}_{8 \text { Test } 13}(\mathrm{RO})=0.2 \mathrm{~m}_{8 \mathrm{Tes} 13}\left(\Theta_{8}\right)=0.8 \\
\mathrm{~m}_{8 \text { Test } 1}(\mathrm{RO})=0.15 \\
\mathrm{~m}_{8 \text { Test } 1}\left(\Theta_{8}\right)=0.85 \\
\mathrm{~m}_{8 \text { Test } 2}(\mathrm{RO})=0.4 \mathrm{~m}_{8 \text { Test } 2}\left(\Theta_{8}\right)=0.6 \\
\mathrm{~m}_{8 \text { Test } 9}(\mathrm{RO})=0.3 \mathrm{~m}_{8 \text { Test } 9}\left(\Theta_{8}\right)=0.7\end{array}$ & none & $\begin{array}{l}\mathrm{m}_{8}^{\mathrm{t}}(\mathrm{RO})=0.85 \\
\mathrm{~m}_{8}^{\mathrm{t}}\left(\Theta_{8}\right)=0.15\end{array}$ \\
\hline 9. & $\begin{array}{l}\Theta_{9}=\{(\mathrm{EO}, \mathrm{RO}), \\
(\mathrm{EO}, \sim \mathrm{RO}), \\
(\sim \mathrm{EO}, \mathrm{RO}), \\
(\sim \mathrm{EO}, \sim \mathrm{RO})\}\end{array}$ & $\mathrm{m}_{9}^{\mathrm{t}}\left(\Theta_{9}\right)=1.0$ & $\begin{array}{l}\mathrm{m}_{8 \Theta 9}(\{(\mathrm{EO}, \mathrm{RO}),(\sim \mathrm{EO}, \\
\mathrm{RO})\})=\mathrm{m}_{8}^{\mathrm{t}}(\mathrm{RO})=0.85 \\
\mathrm{~m}_{8 \Theta 9}\left(\Theta_{9}\right)=\mathrm{m}_{8}^{\mathrm{t}}\left(\Theta_{8}\right)=0.15 \\
\mathrm{~m}_{7 \Theta 9}(\{(\mathrm{EO}, \mathrm{RO}),(\mathrm{EO}, \sim \mathrm{RO})\}) \\
=\mathrm{m}_{7}^{\mathrm{t}}(\mathrm{EO})=0.7 \\
\mathrm{~m}_{7 \Theta 9}\left(\Theta_{9}\right)=\mathrm{m}_{7}^{\mathrm{t}}\left(\Theta_{7}\right)=0.3\end{array}$ & $\begin{array}{l}\mathrm{m}_{9}^{\mathrm{t}}(\mathrm{EO}, \mathrm{RO})=\left(0.85^{*} 0.7\right)=0.595 \\
\mathrm{~m}_{9}^{\mathrm{t}}(\{(\mathrm{EO}, \mathrm{RO}),(\sim \mathrm{EO}, \mathrm{RO})\}) \\
=\left(0.85^{*} 0.3\right)=0.255 \\
\mathrm{~m}_{9}^{\mathrm{t}}(\{(\mathrm{EO}, \mathrm{RO}), \quad(\mathrm{EO}, \quad \sim \mathrm{RO})\}) \\
=\left(0.15^{*} 0.7\right)=0.105 \\
\mathrm{~m}_{9}^{\mathrm{t}}\left(\Theta_{9}\right)=\left(0.15^{*} 0.3\right)=0.045\end{array}$ \\
\hline 10. & $\begin{array}{l}\Theta_{10}{ }^{\# \# \#}=\{(\mathrm{E}, \mathrm{EO}, \mathrm{RO}), \\
(\sim \mathrm{E}, \mathrm{EO}, \sim \mathrm{RO}), \\
(\sim \mathrm{E}, \sim \mathrm{EO}, \mathrm{RO}), \\
(\sim \mathrm{E}, \sim \mathrm{EO}, \sim \mathrm{RO})\})\end{array}$ & $\mathrm{m}_{10}^{\mathrm{t}}\left(\Theta_{10}\right)=1.0$ & $\begin{array}{l}\mathrm{m}_{9 \Theta 10}(\mathrm{E}, \mathrm{EO}, \mathrm{RO})=\mathrm{m}_{9}^{\mathrm{t}}(\mathrm{EO}, \mathrm{RO})=0.595 \\
\mathrm{~m}_{9 \Theta 10}(\{(\mathrm{E}, \mathrm{EO}, \mathrm{RO}),(\sim \mathrm{E}, \sim \mathrm{EO}, \mathrm{RO})\}) \\
=\mathrm{m}_{9}^{\mathrm{t}}(\{(\mathrm{EO}, \mathrm{RO}),(\sim \mathrm{EO}, \mathrm{RO})\})=0.255 \\
\mathrm{~m}_{9 \Theta 10}(\{(\mathrm{E}, \mathrm{EO}, \mathrm{RO}),(\sim \mathrm{E}, \mathrm{EO}, \sim \mathrm{RO})\}) \\
=\mathrm{m}_{9}^{\mathrm{t}}(\{(\mathrm{EO}, \mathrm{RO}),(\mathrm{EO}, \sim \mathrm{RO})\})=0.105 \\
\mathrm{~m}_{9 \Theta 10}\left(\Theta_{10}\right)=\mathrm{m}_{9}\left(\Theta_{9}\right)=0.045\end{array}$ & $\begin{array}{l}\mathrm{m}_{10}^{\mathrm{t}}(\mathrm{E}, \mathrm{EO}, \mathrm{RO})=0.595 \\
\mathrm{~m}_{10}^{\mathrm{t}}(\{(\mathrm{E}, \mathrm{EO}, \mathrm{RO}),(\sim \mathrm{E}, \sim \mathrm{EO}, \mathrm{RO} \\
)\})=0.255 \\
\mathrm{~m}_{10}^{\mathrm{t}}(\{(\mathrm{E}, \mathrm{EO}, \mathrm{RO}),(\sim \mathrm{E}, \\
\mathrm{EO}, \sim \mathrm{RO})\})=0.105 \\
\mathrm{~m}_{10}^{\mathrm{t}}\left(\Theta_{10}\right)=0.045\end{array}$ \\
\hline
\end{tabular}

\#\#\# In general this node contains 8 elements: $\{(\mathrm{E}, \mathrm{EO}, \mathrm{RO}),(\mathrm{E}, \mathrm{EO}, \sim \mathrm{RO}),(\mathrm{E}, \sim \mathrm{EO}, \mathrm{RO}),(\sim \mathrm{E}, \mathrm{EO}, \mathrm{RO}),(\mathrm{E}, \sim \mathrm{EO}, \sim \mathrm{RO}),(\sim \mathrm{E}, \sim \mathrm{EO}, \mathrm{RO}),(\sim \mathrm{E}, \mathrm{EO}, \sim \mathrm{RO})$,

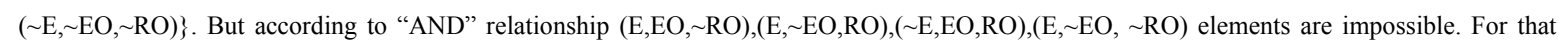
reason the coressponding non zero elements are $\{(\mathrm{E}, \mathrm{EO}, \mathrm{RO}),(\sim \mathrm{E}, \sim \mathrm{EO}, \mathrm{RO}),(\sim \mathrm{E}, \mathrm{EO}, \sim \mathrm{RO}),(\sim \mathrm{E}, \sim \mathrm{EO}, \sim \mathrm{RO})\}$ 
Table 5. Propagation of $\mathrm{m}$ values (belief functions) in the Markov tree in figure 2 for node 11,12 and 13

\begin{tabular}{|c|c|c|c|c|}
\hline Node & Frame of the node & $\begin{array}{c}\text { m-values } \\
\text { defined at the } \\
\text { node }\end{array}$ & $\begin{array}{l}\text { m-values at the node from neighboring } \\
\text { node/nodes }\end{array}$ & $\begin{array}{l}\text { the total } m \text {-values at the node } \\
\text { (obtained from combining m-values in } \\
\text { colums } 3 \text { and } 4 \text { using Dempster's } \\
\text { combination rule) }\end{array}$ \\
\hline 11. & $\begin{array}{l}\Theta_{11}^{+}=\{(\mathrm{E}, \mathrm{EO}, \mathrm{RO}, \mathrm{CP}), \\
(\sim \mathrm{E}, \mathrm{EO}, \mathrm{RO}, \sim \mathrm{CP}), \\
(\sim \mathrm{E}, \mathrm{EO}, \sim \mathrm{RO}, \mathrm{CP}), \\
(\sim \mathrm{E}, \sim \mathrm{EO}, \mathrm{RO}, \mathrm{CP}), \\
(\sim \mathrm{E}, \sim \mathrm{EO}, \sim \mathrm{RO}, \sim \mathrm{CP})\}\end{array}$ & $\mathrm{m}_{11}^{\mathrm{t}}\left(\Theta_{11}\right)=1.0$ & $\begin{array}{l}\mathrm{m}_{10 \Theta 11}(\mathrm{E}, \mathrm{EO}, \mathrm{RO}, \mathrm{CP})=\mathrm{m}_{10}^{\mathrm{t}}(\mathrm{E}, \mathrm{EO}, \mathrm{RO})=0.595 \\
\mathrm{~m}_{10 \Theta 11}(\mathrm{E}, \mathrm{EO}, \mathrm{RO}, \mathrm{CP}),(\sim \mathrm{E}, \sim \mathrm{EO}, \mathrm{RO}, \mathrm{CP})= \\
\left.\left.=\mathrm{m}_{10}^{\mathrm{t}}(\mathrm{E}, \mathrm{EO}, \mathrm{RO}),(\sim \mathrm{E}, \sim \mathrm{EO}, \mathrm{RO})\right\}\right)=0.255 \\
\mathrm{~m}_{10 \Theta 11}(\mathrm{E}, \mathrm{EO}, \mathrm{RO}, \mathrm{CP}),(\sim \mathrm{E}, \mathrm{EO}, \sim \mathrm{RO}, \mathrm{CP}) \\
\left.\left.=\mathrm{m}_{10}^{\mathrm{t}}(\mathrm{E}, \mathrm{EO}, \mathrm{RO}),(\sim \mathrm{E}, \mathrm{EO}, \sim \mathrm{RO})\right\}\right)=0.105 \\
\mathrm{~m}_{10 \Theta 11}=\mathrm{m}_{10}^{\mathrm{t}}\left(\Theta_{10}\right)=0.045 \\
\mathrm{~m}_{12 \Theta 11}(\{(\mathrm{E}, \mathrm{EO}, \mathrm{RO}, \mathrm{CP}),(\sim \mathrm{E}, \mathrm{EO}, \sim \mathrm{RO}, \mathrm{CP}) \\
(\sim \mathrm{E}, \sim \mathrm{EO}, \mathrm{RO}, \mathrm{CP})\})=\mathrm{m}_{12}^{\mathrm{t}}(\mathrm{CP})=0.56 \\
\mathrm{~m}_{12 \Theta 11}=\mathrm{m}_{12}^{\mathrm{t}}\left(\Theta_{12}\right)=0.44\end{array}$ & $\begin{array}{l}\mathrm{m}_{11}^{\mathrm{t}}(\mathrm{E}, \mathrm{EO}, \mathrm{RO}, \mathrm{CP})=(0.595 * 0.56)+ \\
\left((0.595 * 0.44)+\left(0.255^{*} 0.56\right)+\right. \\
(0.105 * 0.44)+\left(0.045^{*} 0.56\right)=0.8092 \\
\mathrm{~m}^{\mathrm{t}}{ }_{11}(\{(\mathrm{E}, \mathrm{EO}, \mathrm{RO}, \mathrm{CP}),(\sim \mathrm{E}, \sim \mathrm{EO}, \mathrm{RO}, \mathrm{CP})\}) \\
=\left(0.255^{*} 0.44\right)=0.1122 \\
\mathrm{~m}_{11}^{\mathrm{t}}(\{(\mathrm{E}, \mathrm{EO}, \mathrm{RO}, \mathrm{CP}),(\sim \mathrm{E}, \mathrm{EO}, \sim \mathrm{RO}, \mathrm{CP})\}) \\
=(0.105 * 0.56)=0.0588 \\
\mathrm{~m}_{11}^{\mathrm{t}}\left(\Theta_{11}\right)=0.045^{*} 0.44=0.0198\end{array}$ \\
\hline 12. & $\Theta_{12}=\{\mathrm{CP}, \sim \mathrm{CP}\}$ & $\begin{array}{l}\mathrm{m}_{12 \text { Test } 1}(\mathrm{CP})=0.2 \\
\mathrm{~m}_{12 \text { Test } 1}\left(\Theta_{7}\right)=0.8 \\
\mathrm{~m}_{12 \text { Test } 2}(\mathrm{CP})=0.2 \\
\mathrm{~m}_{12 \text { Test } 2}\left(\Theta_{7}\right)=0.8 \\
\mathrm{~m}_{12 \text { Test } 9}(\mathrm{CP})=0.3 \\
\mathrm{~m}_{12 \text { Test } 9}\left(\Theta_{7}\right)=0.7\end{array}$ & none & $\begin{array}{l}\mathrm{m}_{12}^{\mathrm{t}}(\mathrm{CP})=0.56 \\
\mathrm{~m}_{12}^{\mathrm{t}}\left(\Theta_{12}\right)=0.44\end{array}$ \\
\hline 13. & $\begin{array}{l}\Theta_{13}=\{(\mathrm{E}, \mathrm{RO}) \\
(\mathrm{E}, \sim \mathrm{RO}) \\
(\sim \mathrm{E}, \mathrm{RO}) \\
(\sim \mathrm{E}, \sim \mathrm{RO})\}\end{array}$ & $\mathrm{m}_{13}\left(\Theta_{13}\right)=1.0$ & $\begin{array}{l}\mathrm{m}_{11}{ }_{13}(\mathrm{E}, \mathrm{RO})=\mathrm{m}_{11}^{\mathrm{t}}(\mathrm{E}, \mathrm{EO}, \mathrm{RO}, \mathrm{CP})=0.8092 \\
\mathrm{~m}_{11 \emptyset 13}(\{(\mathrm{E}, \mathrm{RO}),(\mathrm{E}, \sim \mathrm{RO})\})= \\
\mathrm{m}_{11}^{\mathrm{t}}(\{(\mathrm{E}, \mathrm{EO}, \mathrm{RO}, \mathrm{CP}),(\sim \mathrm{E}, \mathrm{EO}, \sim \mathrm{RO}, \mathrm{CP})\}) \\
=0.1122 \\
\mathrm{~m}_{11 \emptyset 13}(\{(\mathrm{E}, \mathrm{RO}),(\sim \mathrm{E}, \mathrm{RO})\})= \\
\mathrm{m}_{11}^{\mathrm{t}}(\{(\mathrm{E}, \mathrm{EO}, \mathrm{RO}, \mathrm{CP}), \\
(\sim \mathrm{E}, \sim \mathrm{EO}, \mathrm{RO}, \mathrm{CP})\})=0.0588 \\
\mathrm{~m}_{11 \emptyset 13}\left(\Theta_{6}\right)=\mathrm{m}_{11}^{\mathrm{t}}\left(\Theta_{11}\right)=0.0198\end{array}$ & $\begin{array}{l}\mathrm{m}_{13}^{\mathrm{t}}(\mathrm{E}, \mathrm{RO})=0.8092 \\
\mathrm{~m}_{13}^{\mathrm{t}}(\{(\mathrm{E}, \mathrm{RO}),(\mathrm{E}, \sim \mathrm{RO})\})=0.1122 \\
\mathrm{~m}_{13}^{\mathrm{t}}(\{(\mathrm{E}, \mathrm{RO}),(\sim \mathrm{E}, \mathrm{RO})\})=0.0588 \\
\mathrm{~m}_{13}^{\mathrm{t}}\left(\Theta_{13}\right)=0.0198\end{array}$ \\
\hline \multicolumn{5}{|c|}{$\begin{array}{l}+ \text { In general this node contains } 13 \text { elements: }\{(\mathrm{E}, \mathrm{EO}, \mathrm{RO}, \mathrm{CP}),(\mathrm{E}, \mathrm{EO}, \mathrm{RO}, \sim \mathrm{CP}),(\mathrm{E}, \mathrm{EO}, \sim \mathrm{RO}, \mathrm{CP}),(\mathrm{E}, \sim \mathrm{EO}, \mathrm{RO}, \mathrm{CP}),(\sim \mathrm{E}, \mathrm{EO}, \mathrm{RO}, \mathrm{CP}),(\mathrm{E}, \mathrm{EO}, \sim \mathrm{RO} \sim \mathrm{CP}), \\
(\mathrm{E}, \sim \mathrm{EO}, \mathrm{RO}, \sim \mathrm{CP}),(\sim \mathrm{E}, \mathrm{EO}, \mathrm{RO}, \sim \mathrm{CP}),(\mathrm{E}, \sim \mathrm{EO}, \sim \mathrm{RO}, \mathrm{CP}),(\sim \mathrm{E}, \mathrm{EO}, \sim \mathrm{RO}, \mathrm{CP}),(\sim \mathrm{E}, \sim \mathrm{EO}, \mathrm{RO}, \mathrm{CP}),(\sim \mathrm{E}, \sim \mathrm{EO}, \sim \mathrm{RO}, \mathrm{CP}),(\sim \mathrm{E}, \sim \mathrm{EO}, \sim \mathrm{RO}, \sim \mathrm{CP})\} . \mathrm{But} \\
\text { according to "AND" relationship }(\mathrm{E}, \mathrm{EO}, \mathrm{RO}, \sim \mathrm{CP}),(\mathrm{E}, \mathrm{EO}, \sim \mathrm{RO}, \mathrm{CP}),(\mathrm{E}, \sim \mathrm{EO}, \mathrm{RO}, \mathrm{CP}),(\sim \mathrm{E}, \mathrm{EO}, \mathrm{RO}, \mathrm{CP}),(\mathrm{E}, \mathrm{EO}, \sim \mathrm{RO} \sim \mathrm{CP}),(\mathrm{E}, \sim \mathrm{EO}, \mathrm{RO}, \sim \mathrm{CP}),,(\mathrm{E}, \\
\sim \mathrm{EO}, \sim \mathrm{RO}, \mathrm{CP}), \\
(\sim \mathrm{E}, \sim \mathrm{EO}, \sim \mathrm{RO}, \mathrm{CP}) \text { elements are impossible. For that reason the coressponding non zero elements are }\{(\mathrm{E}, \mathrm{EO}, \mathrm{RO}, \mathrm{CP}),(\sim \mathrm{E}, \mathrm{EO}, \mathrm{RO}, \sim \mathrm{CP}),(\sim \mathrm{E}, \\
\mathrm{EO}, \sim \mathrm{RO}, \mathrm{CP}),(\sim \mathrm{E}, \sim \mathrm{EO}, \mathrm{RO}, \mathrm{CP}),(\sim \mathrm{E}, \sim \mathrm{EO}, \sim \mathrm{RO}, \sim \mathrm{CP})\}\end{array}$} \\
\hline
\end{tabular}

Table 6. Propation of $\mathrm{m}$ values (belief functions) in the Markov tree in Figure 2. for node 14 and 15

\begin{tabular}{|c|c|c|c|c|}
\hline Node & $\begin{array}{l}\text { Frame of the } \\
\text { node }\end{array}$ & $\begin{array}{c}\text { m-values } \\
\text { defined at the } \\
\text { node }\end{array}$ & $\begin{array}{l}\text { m-values at the node from neighboring } \\
\text { node/nodes }\end{array}$ & $\begin{array}{l}\text { the total m-values at the node (obtained from } \\
\text { combining m-values in colums } 3 \text { and } 4 \text { using } \\
\text { Dempster's combination rule) }\end{array}$ \\
\hline \multirow[t]{13}{*}{14.} & $\Theta_{14}=(\{(\mathrm{E}, \mathrm{V}, \mathrm{RO})$ & $\mathrm{m}_{14}\left(\Theta_{14}\right)=1.0$ & $\mathrm{~m}_{13 \phi_{14}(\{(\mathrm{E}, \mathrm{V}, \mathrm{RO}),(\mathrm{E}, \sim \mathrm{V}, \mathrm{RO})\})}$ & $\mathrm{m}_{14}^{\mathrm{t}}(\mathrm{E}, \mathrm{V}, \mathrm{RO})=(0.8092 * 0.3796)+(0.8092 * 0.1404)$ \\
\hline & $(\mathrm{E}, \mathrm{V}, \sim \mathrm{RO})$ & & $=\mathrm{m}_{13}^{\mathrm{t}}(\mathrm{E}, \mathrm{RO})=0.8092$ & $+(0.1122 * 0.3796)=0.4633$ \\
\hline & $(\mathrm{E} \sim \mathrm{VRO})$ & & $\mathrm{m}_{13} \emptyset_{14}(\{(\mathrm{E}, \mathrm{V}, \mathrm{RO}),(\mathrm{E}, \sim \mathrm{V}, \mathrm{RO})$ & $\mathrm{m}_{14}^{\mathrm{t}}(\mathrm{E}, \mathrm{V}, \mathrm{RO}),(\mathrm{E}, \sim \mathrm{V}, \mathrm{RO})=(0.8092 * 0.1296)+(0.05$ \\
\hline & & & $(\mathrm{E}, \mathrm{V}, \sim \mathrm{RO})\})$ & $88 * 0.3796)$ \\
\hline & & & $=\mathrm{m}_{13}^{\mathrm{t}}(\{(\mathrm{E}, \mathrm{RO}),(\mathrm{E}, \sim \mathrm{RO})\})=0.1122$ & $+(0.8092 * 0.3504)+(0.0198 * 0.3796)=0.4183$ \\
\hline & & & $\mathrm{m}_{13 \emptyset_{14}}(\{(\mathrm{E}, \mathrm{V}, \mathrm{RO}),(\mathrm{E}, \sim \mathrm{V}, \mathrm{RO}),(\sim \mathrm{E}, \mathrm{V}, \mathrm{RO})$ & $\mathrm{m}_{14}^{\mathrm{t}}(\mathrm{E}, \mathrm{V}, \mathrm{RO}),(\mathrm{E}, \sim \mathrm{V}, \mathrm{RO})$ \\
\hline & $(\sim \mathrm{E}, \mathrm{V}, \sim \mathrm{RO})$ & & $(\sim \mathrm{E}, \sim \mathrm{V}, \mathrm{RO})\})=$ & $(\mathrm{E}, \mathrm{V}, \sim \mathrm{RO})=(0.1122 * 0.3504)+(0.1122 * 0.1296)$ \\
\hline & $(\sim \mathrm{E}, \sim \mathrm{V}, \mathrm{RO})$ & & $\mathrm{m}_{13}^{\mathrm{t}}(\{(\mathrm{E}, \mathrm{RO}),(\sim \mathrm{E}, \mathrm{RO})\})=0.0588$ & $=0.0539$ \\
\hline & $(\sim \mathrm{E}, \sim \mathrm{V}, \sim \mathrm{RO})\})$ & & $\mathrm{m}_{13 \emptyset_{14}}=\mathrm{m}_{13}^{\mathrm{t}}\left(\Theta_{13}\right)=0.0198$ & $\mathrm{~m}_{14}^{\mathrm{t}}(\mathrm{E}, \mathrm{V}, \mathrm{RO}),(\mathrm{E}, \sim \mathrm{V}, \mathrm{RO})$ \\
\hline & & & $\mathrm{m}_{6} \emptyset_{14}(\{(\mathrm{E}, \mathrm{V}, \mathrm{RO}),(\mathrm{E}, \sim \mathrm{V}, \mathrm{RO})\})$ & $(\sim \mathrm{E}, \mathrm{V}, \mathrm{RO}),(\sim \mathrm{E}, \sim \mathrm{V}, \mathrm{RO})=(0.0588 * 0.1296)=0.0076$ \\
\hline & & & $=\mathrm{m}_{6}^{\mathrm{t}}(\mathrm{V}, \mathrm{RO})=0.3796$ & $\mathrm{~m}_{14}^{\mathrm{t}}(\mathrm{E}, \mathrm{V}, \mathrm{RO}),(\mathrm{E}, \sim \mathrm{V}, \mathrm{RO})$ \\
\hline & & & $\mathrm{m}_{6 \emptyset_{14}}(\{(\mathrm{E}, \mathrm{V}, \mathrm{RO}),(\mathrm{E}, \sim \mathrm{V}, \mathrm{RO}),(\mathrm{E}$, & $(\mathrm{E}, \sim \mathrm{V}, \mathrm{RO}),(\sim \mathrm{E}, \sim \mathrm{V}, \mathrm{RO})=(0.1122 * 0.1404)+$ \\
\hline & & & $\sim \mathrm{V}, \mathrm{RO}),(\sim \mathrm{E}, \sim \mathrm{V}, \mathrm{RO})\})=\mathrm{m}_{6}^{\mathrm{t}}(\{(\mathrm{V}, \mathrm{RO})$, & $(0.0198 * 0.1404)=0.0185$ \\
\hline
\end{tabular}




\begin{tabular}{|c|c|c|c|c|}
\hline & & & $\begin{array}{l}(\sim \mathrm{V}, \mathrm{RO})\})=0.1404 \\
\mathrm{~m}_{6} \oplus_{14}(\{(\mathrm{E}, \mathrm{V}, \mathrm{RO}),(\sim \mathrm{E}, \mathrm{V}, \mathrm{RO}), \\
(\mathrm{E}, \sim \mathrm{V}, \sim \mathrm{RO}), \\
(\sim \mathrm{E}, \sim \mathrm{V}, \sim \mathrm{RO})\})=\mathrm{m}_{6}^{\mathrm{t}}(\{(\mathrm{V}, \mathrm{RO}), \\
(\sim \mathrm{V}, \sim \mathrm{RO})\})=0.3504 \\
\mathrm{~m}_{6 \oplus_{14}}=\mathrm{m}_{3}^{\mathrm{t}}\left(\Theta_{3}\right)=0.1296\end{array}$ & $\begin{array}{l}\mathrm{m}_{14}^{\mathrm{t}}(\mathrm{E}, \mathrm{V}, \mathrm{RO}),(\sim \mathrm{E}, \mathrm{V}, \mathrm{RO}),(\mathrm{E}, \sim \mathrm{V}, \sim \mathrm{RO}),(\sim \mathrm{E}, \sim \mathrm{V}, \sim \mathrm{R} \\
\mathrm{O})=(0.0198 * 0.3504)=0.0069 \\
\mathrm{~m}^{\mathrm{t}}{ }_{14}(\mathrm{E}, \mathrm{V}, \mathrm{RO}),(\sim \mathrm{E}, \mathrm{V}, \mathrm{RO})=(0.0588 * 0.3504)=0.020 \\
6 \\
\mathrm{~m}^{\mathrm{t}}{ }_{14}(\mathrm{E}, \mathrm{V}, \mathrm{RO}),(\sim \mathrm{E}, \sim \mathrm{V}, \mathrm{RO}) \\
=(0.0588 * 0.1404)=0.0083 \\
\mathrm{~m}^{\mathrm{t}}{ }_{14}\left(\Theta_{14}\right)=(0.0198 * 0.1296)=0.0026\end{array}$ \\
\hline 15. & $\begin{array}{l}\Theta_{15}=\{(\mathrm{E}, \mathrm{V}) \\
(\mathrm{E}, \sim \mathrm{V}) \\
(\sim \mathrm{E}, \mathrm{V}) \\
(\sim \mathrm{E}, \sim \mathrm{V})\}\end{array}$ & $\mathrm{m}_{15}\left(\Theta_{15}\right)=1.0$ & 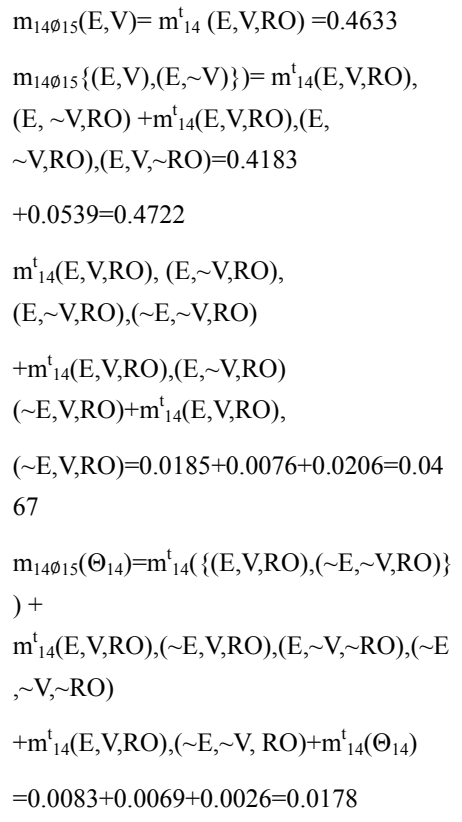 & $\begin{array}{l}\mathrm{m}_{15}^{\mathrm{t}}(\mathrm{E}, \mathrm{V})=0.4633 \\
\mathrm{~m}_{15}^{\mathrm{t}}(\mathrm{E}, \mathrm{V}),(\mathrm{E}, \sim \mathrm{V})=0.4722 \\
\mathrm{~m}_{15}^{\mathrm{t}}(\mathrm{E}, \mathrm{V}),(\sim \mathrm{E}, \mathrm{V})=0.0467 \\
\mathrm{~m}_{15}^{\mathrm{t}}\left(\Theta_{15}\right)=0.0178\end{array}$ \\
\hline
\end{tabular}

Table 7. Propagation of $m$ values (belief functions) in the Markov tree in Figure 2. for node 16 and 17

\begin{tabular}{|c|c|c|c|c|}
\hline Node & $\begin{array}{c}\text { Frame of the } \\
\text { node }\end{array}$ & $\begin{array}{l}\text { m-values defined } \\
\text { at the node }\end{array}$ & m-values at the node from neighboring node/nodes & $\begin{array}{l}\text { the total m-values at the node } \\
\text { (obtained from combining } \\
\text { m-values in colums } 3 \text { and } 4 \text { using } \\
\text { Dempster's combination rule) }\end{array}$ \\
\hline \multirow[t]{4}{*}{16.} & $\Theta_{16}{ }^{++}=\{(\mathrm{I}, \mathrm{E}, \mathrm{V})$ & $\mathrm{m}_{16}\left(\Theta_{16}\right)=1.0$ & 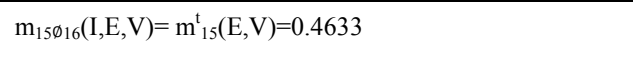 & $\mathrm{m}_{16}^{\mathrm{t}}(\mathrm{I}, \mathrm{E}, \mathrm{V})=0.4633$ \\
\hline & $(\sim \mathrm{I}, \mathrm{E}, \sim \mathrm{V})$ & & $\mathrm{m}_{15 \oplus 16}(\{(\mathrm{I}, \mathrm{E}, \mathrm{V}),(\sim \mathrm{I}, \mathrm{E}, \sim \mathrm{V})\})=\mathrm{m}_{15}^{\mathrm{t}}{ }_{15}(\mathrm{E}, \mathrm{V}),(\mathrm{E}, \sim \mathrm{V})=0.4722$ & $\mathrm{~m}_{16}^{\mathrm{t}}(\{(\mathrm{I}, \mathrm{E}, \mathrm{V}),(\sim \mathrm{I}, \mathrm{E}, \sim \mathrm{V})\})=0.04722$ \\
\hline & $(\sim \mathrm{I}, \sim \mathrm{E}, \mathrm{V})$ & & $\mathrm{m}_{15} \oplus_{16}(\{(\mathrm{I}, \mathrm{E}, \mathrm{V}),(\sim \mathrm{I}, \sim \mathrm{E}, \mathrm{V})\})=\mathrm{m}_{15}^{\mathrm{t}}(\mathrm{E}, \mathrm{V}),(\sim \mathrm{E}, \mathrm{V})=0.0467$ & $\mathrm{~m}_{16}^{\mathrm{t}}(\{(\mathrm{I}, \mathrm{E}, \mathrm{V}),(\sim \mathrm{I}, \sim \mathrm{E}, \mathrm{V})\})=0.0467$ \\
\hline & $(\sim \mathrm{I}, \sim \mathrm{E}, \sim \mathrm{V})\}$ & & 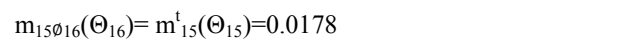 & $\mathrm{m}_{16}^{\mathrm{t}}\left(\Theta_{16}\right)=0.0178$ \\
\hline \multirow[t]{4}{*}{17.} & $\Theta_{17}\{\mathrm{I}, \sim \mathrm{I}\}$ & $\mathrm{m}_{17 \text { Add.Infor. }}(\mathrm{I})=0.6$ & 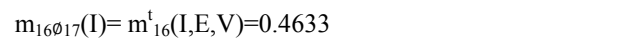 & $\mathrm{m}_{17}^{\mathrm{t}}(\mathrm{I})=0.79$ \\
\hline & & $\mathrm{m}_{17 \text { Add.Infor. }}\left(\Theta_{17}\right)=0$ & 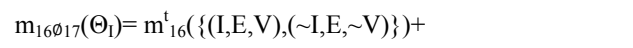 & $\mathrm{m}_{17}^{\mathrm{t}}\left(\Theta_{17}\right)=0.21$ \\
\hline & & 4 & $\mathrm{~m}_{16}^{\mathrm{t}}(\{(\mathrm{I}, \mathrm{E}, \mathrm{V}),(\sim \mathrm{I}, \sim \mathrm{E}, \mathrm{V})\})+$ & \\
\hline & & & $\mathrm{m}_{16}^{\mathrm{t}}\left(\Theta_{16}\right)=0.4722+0.0467+0.0178=0.5367$ & \\
\hline $\begin{array}{l}\text { relati } \\
(\sim \mathrm{I}, \sim\end{array}$ & $\begin{array}{l}\text { ral this node } \\
(\mathrm{I}, \mathrm{E}, \sim \mathrm{V}),(\mathrm{I}, \\
\sim \mathrm{I}, \mathrm{E}, \sim \mathrm{V}),(\sim \mathrm{I},\end{array}$ & $\begin{array}{l}\text { ins } 8 \text { elements: }\{0 \\
),(\sim \mathrm{I}, \mathrm{E}, \mathrm{V}),(\mathrm{I}, \sim \mathrm{E} \text {, } \\
\mathrm{V})\}\end{array}$ & $\begin{array}{l}\mathrm{V}),(\mathrm{I}, \mathrm{E}, \sim \mathrm{V}),(\mathrm{I}, \sim \mathrm{E}, \mathrm{V}),(\sim \mathrm{I}, \mathrm{E}, \mathrm{V}),(\mathrm{I}, \sim \mathrm{E}, \sim \mathrm{V}),(\sim \mathrm{I}, \sim \mathrm{E}, \mathrm{V}),(\sim \mathrm{I}, \mathrm{E}, \sim \\
\text { elements are impossible. For that reason the coressp }\end{array}$ & $\begin{array}{l}\sim \mathrm{I}, \sim \mathrm{E}, \sim \mathrm{V})\} . \text { But according to "AND" } \\
\text { ng non zero elements are }\{(\mathrm{I}, \mathrm{E}, \mathrm{V})\end{array}$ \\
\hline
\end{tabular}


Table 8. The audit procedures used in Figure 1

\begin{tabular}{|c|c|c|}
\hline $\begin{array}{c}\text { Audit } \\
\text { Procedure }\end{array}$ & $\begin{array}{l}\text { Audit } \\
\text { Test } \\
\text { Number }\end{array}$ & Inventory Account Procedures \\
\hline $\mathrm{B}$ & 1. & Monitoring the client's physical inventory. \\
\hline $\mathrm{B}$ & 2. & $\begin{array}{l}\text { Discuss the procedures of inventory valuation which are applied by the client for identify; } \\
\text { - any alterations in particular products, } \\
\text { - any alterations in production procedures, } \\
\text { - any alterations in accounting and/or cost accounting policies and their effects on inventory valuation } \\
\text { - observation of methods which are aggregate inventory items' cost, } \\
\text { - observation of client's inventory pricing policies and procedures } \\
\text { - observation of physical conditions of inventory }\end{array}$ \\
\hline A & 3. & $\begin{array}{l}\text { Investigate the clients' clerical correctness of the physical inventory summary; } \\
\text {-make a trace test for inventory summary } \\
\text {-make a comparison between the tag or count sheet check numbers acquired during the observation to these } \\
\text { employed to gather the inventory summary } \\
\text {-examine any tags or count sheets added or removed } \\
\text { - reconcile the physical inventory summary to the general ledger account balance } \\
\text {-examine and state critical reconciling items }\end{array}$ \\
\hline A & 4. & $\begin{array}{l}\text { Revise the physical inventory list and decide individually important items of raw materials, work-in-process } \\
\text { and finished products; } \\
\text {-determine individually sinificant amounts } \\
\text {-review prior history of costing errors and misstatements } \\
\text {-review SAS No.96 audit documentation requires }\end{array}$ \\
\hline $\mathrm{D}$ & 5. & $\begin{array}{l}\text { Test the cost of products and raw materials; } \\
\text { - to the extent considered necessary, vouch the cost to the most recent vendor invoices for the period of the } \\
\text { audit } \\
\text { - decide if freight discounts and allowances are steadily handled when calculating the cost of the inventory } \\
\text {-investigate similar products codes } \\
\text {-relate the costs of other untested items to prices used for the same item in prior years. } \\
\text {-enquire if future purchase costs of any significant products will be decreased. Shortly test replacement costs } \\
\text { on the items tested by investigating current purchase price list or invoices for purchases after year end } \\
\text {-evaluate the net realizable value of purchased finished goods by reference to current sales prices for the } \\
\text { products (disposition cost should be deducted from the sales price before making the comparison to cost) } \\
\text { - decide if the valuation method is in accordance with GAAP, consistently applied. }\end{array}$ \\
\hline $\mathrm{D}$ & 6. & $\begin{array}{l}\text { Test the costs; } \\
\text {-determine the cost accounting system, a job order system or process cost system. (decide the source } \\
\text { documents employed to calculate finished goods costs) } \\
\text {-for finished goods picked for testing acquire a copy of a recent job order or process production report for the } \\
\text { produce of the item } \\
\text {-investigate quantities of raw material and labor charged during manufacturing to pertinent supporting } \\
\text { documents such as bills of material, requisition forms, time cards, etc } \\
\text {-confirm the cost of material to the raw material inventory summary or to vendor invoices } \\
\text {-confirm the labor hours and rates to payroll records. }\end{array}$ \\
\hline $\mathrm{D}$ & 7. & $\begin{array}{l}\text { Test the pricing of inventory; } \\
\text { - document the sampling plan, comprising the items chosen. } \\
\text { - assess the outcomes and project the misstatement }\end{array}$ \\
\hline $\mathrm{D}$ & 8. & $\begin{array}{l}\text { Decide whether allowances have been made for scrap, obsolete, unsalable, slow-moving, or overstocked } \\
\text { items; } \\
\text {-decide the client's procedure for identifying potential problems } \\
\text { - compare the observation of physical inventory count to the final inventory listing } \\
\text {-review perpetual records, sales analyses, and other information to determine actual usage of the items during } \\
\text { the year } \\
\text {-review old or inactive jobs and determine whether individual items are properly valued based upon potential } \\
\text { market value. } \\
\text {-compare the prior year listing of obsolete items to the final inventory listing of the current year to decide that } \\
\text { prior year valuations have not been rised. } \\
\text {-examine and interpret any unusual exceptions }\end{array}$ \\
\hline B & 9. & $\begin{array}{l}\text { Trace all shipping and receiving transactions selected for testing during the inventory observation to the } \\
\text { appropriate journals or detail lists of accounts payable }\end{array}$ \\
\hline
\end{tabular}




\begin{tabular}{|c|c|c|}
\hline & & -determine that these transactions are reported appropriately in the period to which they apply \\
\hline $\mathrm{C}$ & 10 . & $\begin{array}{l}\text { From a review of bank confirmations, debt confirmations, directors' minutes and inquiry of management, } \\
\text { decide whether any inventory has been pledged or assigned to others to collateralize debt. Sum up any such } \\
\text { situations for disclosure. }\end{array}$ \\
\hline $\mathrm{C}$ & 11. & $\begin{array}{l}\text { The determination to employ supplement procedures should be based on a consideration of whether } \\
\text { information acquired or misstatements identified by performing substantive tests or from other sources during } \\
\text { the audit alter your judgement about the need to acquire a further understanding of control activities, the } \\
\text { assessed level of risk of material misstatements (whether caused by error or fraud), and on an evaluation of } \\
\text { whether the basic procedures have been adequate to accomplish the audit objectives. Attach audit program } \\
\text { sheets to document supplement procedures; } \\
\text { - intercompany profit } \\
\text {-retail inventory method } \\
\text {-LIFO inventory method. } \\
\text {-inventory tested at an interim date. }\end{array}$ \\
\hline $\mathrm{C}$ & 12. & $\begin{array}{l}\text { Take into account whether procedures performed are sufficient to reply to identified fraud risk factors; } \\
\text { - Specific replies to identified fraud risk factors are addressed in individual audit programs }\end{array}$ \\
\hline $\mathrm{C}$ & 13. & $\begin{array}{l}\text { Take into account whether the results of audit procedures demonstrate reportable conditions in internal control } \\
\text { and, if so, add to the memo of points for the communication of reportable conditions }\end{array}$ \\
\hline
\end{tabular}

Source: http://www.acsu.buffalo.edu/ yushaoch/inventory\%20program.doc.

\section{Discussion}

In this study, we illustrated the aggregation of the audit evidence based on belief function approach for inventory account auditing and we discussed a general scheme for aggregating information that were presented by audit evidence. For this purpose, we used a real audit case's file data in Turkey. We reviewed auditors' works and created an evidential network for propagating beliefs. We followed three steps in this study. The first step deals with identifiying the variables based on audit files. The second step deals with designing a Markov Tree which represents propagating $\mathrm{m}$ values. The third step deals with the implemantation of data and comparison of the results. We discussed our results with auditors and we reviewed auditors' report which gives auditors' professional judgements. As a result of this study, the auditors accepted our results which reflect their audit opinions. We also argued how the auditors can use our evidential network for audit planning and how the auditors can use our study to achieve their confidence level's target for the audit risk.

In general, the organization of audit evidence can be illustrated by a network. In this network each audit evidence reflects the level of supports on one objective of an account. The overall belief that an audit objective reflects auditors' professional judgement for an account or financial statements. In other words, we can use belief function framework as a helpful tool in audit works. Using of a belief function approach in audit may make the audit process more effective. We believe that our evidential scheme will provide more effective and efficient audit opportunity and our scheme makes a valuable contribution to the auditing literature.

In this study, we have several limitations for simplicity. For example, we didn't use mixed evidence which represents different contrary level of supports for different management assertions at the same time. We used Dempster's combination rule. We assumed all audit objectives have equal importance. Since assigning different amounts of evidence to support their audit objectives is another study scope, researchers may study on this subject in their future studies. We followed the audit file and examined a manufacturing company's accounts. However, these ones may not be true in auditing context. For example, the auditors can assign more complex $\mathrm{m}$ values for using of mixed evidence. We know Dempster rule is an ineffective way for conflict of audit evidence. In real world situation, an audit aim can be more significant than another audit aim/aims. We have not discussed the appropriateness of audit procedures that auditors applied and different company structures that may have different characteristics that may effect evidential network.

\section{References}

American Institute of Certified Public Accountants. (AICPA) (1983). Statements on Auditing Standarts. Number 47. Retrieved from http://www.aicpa.org/research/standards/auditattest/pages/sas.aspx

Akresh, A. D., Loebbecke, J. K., \& Scott, W. R. (1988). Audit Approaches and Techniques. In A. R. Abdelkhalik \& I. Solomon Research (Eds.), Opportunities in Auditing: The Second Decade (pp. 13-55). American Accounting Association.

Desai, V., Roberts, R., \& Srivastava, R. P. (2010). A Conceptual Model for External Auditor Evaluation of the 
Internal Audit Function Using Belief Functions. Contemporary Accounting Research, 27(2), 537-55. http://dx.doi.org/10.1111/j.1911-3846.2010.01016.x

Fukukawa, H., Mock, T. J., \& Srivastava, R. P. (2014). Assessing the Risk of Fraud at Olympus and Identifying an Effective Audit Plan. The Japanese Accounting Review. http://dx.doi.org/10.11640/tjar.4.2014.01

Gao, L., \& Srivastava, R. P. (2011). The Anatomy of Management Fraud Schemes: Analyses and Implications. Indian Accounting Review, 15(1), 1-23.

Kong, A. (1986). Multivariate belief functions and graphical models. Doctoral Dissertation, Harvard University.

Leslie, D. A. (1984). An Analysis of the Audit Framework Focusing on Inherent Risk and the Role os Statistical Sampling in Compliance Testing. Proceedings of the 1984 Touche Ross/University of Kansas Symposium on Auditing Problems, May, 89-125.

Mock, T., Sun, L., Srivastava, R. P., \& Vasarhelyi, M. (2009). An Evidential Reasoning Approach to Sarbanes-Oxley Mandated Internal Control Risk Assessment under Dempster-Shafer Theory. International Journal of Accounting Systems, 10(1), 65-78. http://dx.doi.org/10.1016/j.accinf.2008.10.003

Shafer, G. A. (1976). Mathematical Theory of Evidence. New Jersey: Princeton University Press.

Shenoy, P. P., \& Shafer, G. (1991). Valuation-Based System for Discrete Optimization. In P. P. Bonissone, M. Henrion, L. N. Kanal, \& J. Lemmer (Eds.), Uncertainty in Artificial Intelligence (Vol. 6, pp. 385-400). Cornell University. http://arxiv.org/abs/1304.1121

Shenoy, P., \& Shenoy, C. (2002). Modeling Financial Portfolios Using Belief Functions. In R. P. Srivastava \& T. J. Mock (Eds.), Belief Functions in Business Decisions, Studies in Fuzziness and Soft Computing (Vol. 88, pp. 316-332). Physica-Verlag. http://hdl.handle.net/1808/158

Srivastava, R. P., \& Shafer, G. (1992). Belief-Function Formulas for Audit Risk. The Accounting Review, 67(2), 249-83. http://www.jstor.org/stable/247724

Srivastava, R. P. (1995). A General Scheme for Aggregating Evidence in Auditing:Propagation of Beliefs in Networks. In A. Miklos \& Vasarhelyi (Eds.), Artificial Intelligence in Accounting and Auditing (Vol. 3, pp. 55-99). Princeton: Markus Wiener Publishers.

Srivastava, R. P., Shenoy, P. P., \& Shafer, G. (1995). Propagating Beliefs in an AND-trees. International Journal of Intelligent Systems, 10(7), 647-664. http://dx.doi.org/10.1002/int.4550100703

Srivastava, R. P. (1997). Audit Decisions Using Belief Functions: A Review. Control and Cybernetics, 26(2), 135-160. http://eycarat.faculty.ku.edu//myssi/publications/

Srivastava, R. P., \& Liu, L. (2003). Applications of Belief Functions in Business Decisions: A Review. Information Systems Frontiers, 5(4), 359-378. http://dx.doi.org/10.1023/B:ISFI.0000005651.93751.4b

Srivastava, R. P., Mock, T. J., \& Turner, J. (2009). Bayesian and Belief Functions Formulas for Auditor Independence Risk Assessment. International Journal of Auditing, 13(3), 163-183. http://dx.doi.org/10.1111/j.1099-1123.2009.00384.x

Srivastava, R. P., Mock, T. J., \& Gao, L. (2011). The Dempster-Shafer Theory of Belief Functions for Managing Uncertainties: An Introduction and Fraud Risk Assessment Illustration. Australian Accounting Review, 21(3), 282-291. http://dx.doi.org/10.1111/j.1835-2561.2011.00135.x

Srivastava, R. P., Mock, T. J., Pincus, K., \& Wright, A. (2012). Causal inference in auditing: A framework. Auditing: A Journal of Practice and Theory, 31(3), 177-201. http://dx.doi.org/10.2308/ajpt-10293

\section{Copyrights}

Copyright for this article is retained by the author(s), with first publication rights granted to the journal.

This is an open-access article distributed under the terms and conditions of the Creative Commons Attribution license (http://creativecommons.org/licenses/by/3.0/). 